\title{
STABILIZATION OF TIMOSHENKO BEAM BY MEANS OF POINTWISE CONTROLS *
}

\author{
Gen-Qi Xu ${ }^{1}$ And Siu Pang Yung ${ }^{2}$
}

\begin{abstract}
We intend to conduct a fairly complete study on Timoshenko beams with pointwise feedback controls and seek to obtain information about the eigenvalues, eigenfunctions, Riesz-BasisProperty, spectrum-determined-growth-condition, energy decay rate and various stabilities for the beams. One major difficulty of the present problem is the non-simplicity of the eigenvalues. In fact, we shall indicate in this paper situations where the multiplicity of the eigenvalues is at least two. We build all the above-mentioned results from an effective asymptotic analysis on both the eigenvalues and the eigenfunctions, and conclude with the Riesz-Basis-Property and the spectrum-determined-growthcondition. Finally, these results are used to examine the stability effects on the system by the location of the pointwise control relative to the length of the whole beam.
\end{abstract}

Mathematics Subject Classification. 34K35, 47A65, 93B52, 93C20.

Received January 18, 2002. Revised April 15, 2003.

\section{INTRODUCTION}

The pointwise feedback control stabilization for Euler-Bernoulli beams, or equivalently stabilization of serially connected beams with dissipative joints, has been widely studied and many results have been obtained (see, for example [1-7] and the references therein). As for Timoshenko beams, the most complete physical model for thick beams, relatively few results are on pointwise feedback controls when comparing with the corresponding boundary feedback control problems [8-16]. This may be due to the complicated changes of the eigenfrequencies arisen from the present of the pointwise feedback control. It is the aim of this paper to carry out a thorough study for this problem and we have obtained asymptotic information for the eigenvalues, the eigenfunctions, the Riesz-Basis-Property, the spectrum-determined-growth-condition and their consequences on stabilities. Our approach is simple but effective. A major step is to conduct a complete asymptotic analysis on the eigenvalues and the eigenfunctions. These then form a very efficient building block for us to deduce all the required results.

Keywords and phrases. Timoshenko beam, pointwise feedback control, generalized eigenfunction system, Riesz basis.

* This research was supported by the Natural Science Foundation of Shan Xi Province in China and a HKRGC grant of code HKU 7133/02P.

${ }^{1}$ Department of Mathematics of Shanxi University, TaiYuan 030006, P.R. China.; e-mail: gqxu@mail.sxu.edu.cn

2 Department of Mathematics of the University of Hong Kong, Hong Kong, P.R. China; e-mail: spyung@hku.hk 
The Timoshenko beam problem with pointwise control that we shall consider is:

$$
\left\{\begin{array}{l}
\rho \ddot{w}(x, t)-\kappa\left(w^{\prime \prime}(x, t)-\varphi^{\prime}(x, t)\right)+u_{1}(t) \delta_{\xi}=0,0<x<\ell, \\
I_{\rho} \ddot{\varphi}(x, t)-E I \varphi^{\prime \prime}(x, t)-\kappa\left(w^{\prime}(x, t)-\varphi(x, t)\right)+u_{2}(t) \delta_{\xi}=0,0<x<\ell, \\
w(0, t)=0, \quad \varphi(0, t)=0, \\
\kappa\left(w^{\prime}(\ell, t)-\varphi(\ell, t)\right)=0, \quad E I \varphi^{\prime}(\ell, t)=0,
\end{array}\right.
$$

where $w(x, t)$ is the deflection of the beam from its equilibrium and $\varphi(x, t)$ is the rotation angle of a filament of the beam at $x$. Here and henceforth the dot and the prime denote derivatives with respect to time and space variables respectively. Also, $I_{\rho}, \rho, E I, \kappa, \ell, u_{1}$ and $u_{2}$ are respectively the mass moment of inertia, mass density, rigidity coefficient, shear modulus of elasticity, length of the beam, external force and external moment. We use $\delta_{\xi}$ to denote a Dirac mass concentrated at the point $\xi \in(0, \ell)$ and consider the following velocity and angular velocity feedback control at point $\xi$ :

$$
\left\{\begin{array}{l}
u_{1}(t)=\alpha \dot{w}(\xi, t) \\
u_{2}(t)=\beta \dot{\varphi}(\xi, t)
\end{array}\right.
$$

where $\alpha$ and $\beta$ are some positive feedback gain constants that can be tuned.

It is the closed loop system (1.1) and (1.2) that we will conduct our research on. The content of this paper is organized as follows. First, we exhibit some elementary properties of the operator $\mathcal{A}$ determined by the closed loop system in Section 2, and then in Section 3, we perform a detail asymptotic analysis for its eigenvalues and eigenfunctions. In Section 4, we apply these asymptotic results to deduce the Riesz basis property for the generalized eigenfunctions of $\mathcal{A}$. Finally in Section 5 , we examine various stabilities of the beams.

\section{BASIC PROPERTIES FOR THE OPERATOR $\mathcal{A}$ OF THE ClOSED LOOP SYSTEM}

In this section, we shall establish some basic properties for the closed loop system $(1.1,1.2)$. To begin, we choose the state space $\mathcal{H}$ to be:

$$
\mathcal{H}:=V_{0}^{1} \times L^{2}(0, \ell) \times V_{0}^{1} \times L^{2}(0, \ell),
$$

where $V_{0}^{k}:=\left\{\varphi \in H^{k}(0, \ell) \mid \varphi(0)=0\right\}, k=1,2$, with $H^{k}(0, \ell)$ being the usual Sobolev space of order $k$. For $Y_{1}:=\left[w_{1}, z_{1}, \varphi_{1}, \psi_{1}\right]^{T}$ and $Y_{2}:=\left[w_{2}, z_{2}, \varphi_{2}, \psi_{2}\right]^{T} \in \mathcal{H}$, in here the superscript $T$ denotes the transposition, the inner product of $\mathcal{H}$ is defined as

$$
\begin{aligned}
\left\langle Y_{1}, Y_{2}\right\rangle:= & \int_{0}^{\ell} \kappa\left(w_{1}^{\prime}(x)-\varphi_{1}(x)\right) \overline{\left(w_{2}^{\prime}(x)-\varphi_{2}(x)\right)} \mathrm{d} x+\int_{0}^{\ell} \rho z_{1}(x) \overline{z_{2}(x)} \mathrm{d} x \\
& +\int_{0}^{\ell} E I \varphi_{1}^{\prime}(x) \overline{\varphi_{2}^{\prime}(x)} \mathrm{d} x+\int_{0}^{\ell} I_{\rho} \psi_{1}(x) \overline{\psi_{2}(x)} \mathrm{d} x
\end{aligned}
$$

and the corresponding norm is denoted by $\|\cdot\|$. If we define operator $\mathcal{A}$ in $\mathcal{H}$ by

$$
\mathcal{A}\left(\begin{array}{c}
w \\
z \\
\varphi \\
\psi
\end{array}\right):=\left(\begin{array}{c}
z \\
\frac{\kappa}{\rho}\left(w^{\prime \prime}-\varphi^{\prime}\right) \\
\psi \\
\frac{E I}{I_{\rho}} \varphi^{\prime \prime}+\frac{\kappa}{I_{\rho}}\left(w^{\prime}-\varphi\right)
\end{array}\right)
$$


with domain

$$
\begin{aligned}
& \mathcal{D}(\mathcal{A}):=\left\{Y=[w, z, \varphi, \psi]^{T} \in \mathcal{H} \mid w, \varphi \in V_{0}^{2}(0, \xi) \cup H^{2}(\xi, \ell), \quad z, \psi \in V_{0}^{1}(0, \ell)\right. \\
& \\
& \left.\kappa\left(w^{\prime}\left(\xi_{+}\right)-w^{\prime}\left(\xi_{-}\right)\right)=\alpha z(\xi), \quad \begin{array}{ll}
E I\left(\varphi^{\prime}\left(\xi_{+}\right)-\varphi^{\prime}\left(\xi_{-}\right)\right)=\beta \psi(\xi), \\
\kappa\left(w^{\prime}(\ell)-\varphi(\ell)\right)=0, & E I \varphi^{\prime}(\ell)=0
\end{array}\right\},
\end{aligned}
$$

then the closed loop system $(1.1,1.2)$ can be written as an evolutionary equation in $\mathcal{H}$ :

$$
\frac{\mathrm{d}}{\mathrm{d} t} Y(t)=\mathcal{A} Y(t), \quad \forall t>0
$$

where

$$
Y(t):=[w(\cdot, t), \dot{w}(\cdot, t), \varphi(\cdot, t), \dot{\varphi}(\cdot, t)]^{T} .
$$

If $(w(t, x), \varphi(x, t))$ is a solution pair for $(1.1,1.2)$, then the energy of the closed loop system is given by

$$
E(t):=\frac{1}{2}\|Y(t)\|^{2}=\frac{1}{2} \int_{0}^{\ell}\left(\kappa\left|w^{\prime}(x, t)-\varphi(x, t)\right|^{2}+\rho|\dot{w}(x, t)|^{2}+E I\left|\varphi^{\prime}(x, t)\right|^{2}+I_{\rho}|\dot{\varphi}(x, t)|^{2}\right) \mathrm{d} x .
$$

Theorem 2.1. The operator $\mathcal{A}$ is dissipative with compact resolvents and generates a $C_{0}$ semigroup of contractions. As a result, the energy of the system $(1.1,1.2)$ is non-increasing in time.

Proof. To show that $\mathcal{A}$ has compact resolvents, it suffices to show that $0 \in \rho(\mathcal{A})$ because for then $\mathcal{A}^{-1}$ will be compact due to the Sobolev Embedding theorem and so are the resolvents of $\mathcal{A}$. So for $F:=\left[f_{1}, f_{2}, g_{1}, g_{2}\right]^{T} \in \mathcal{H}$, we need to find a unique $Y:=[w, z, \varphi, \psi]^{T} \in \mathcal{D}(\mathcal{A})$ such that $\mathcal{A} Y=F$, i.e.,

$$
\left\{\begin{array}{l}
z=f_{1} \\
\frac{\kappa}{\rho}\left(w^{\prime \prime}-\varphi^{\prime}\right)=f_{2} \\
\psi=g_{1} \\
\frac{E I}{I_{\rho}} \varphi^{\prime \prime}+\frac{\kappa}{I_{\rho}}\left(w^{\prime}-\varphi\right)=g_{2}
\end{array}\right.
$$

with conditions

$$
\left\{\begin{array}{l}
w(0)=0, \quad \varphi(0)=0, \quad w\left(\xi_{+}\right)=w\left(\xi_{-}\right), \quad \varphi\left(\xi_{+}\right)=\varphi\left(\xi_{-}\right) \\
\kappa\left(w^{\prime}\left(\xi_{+}\right)-w^{\prime}\left(\xi_{-}\right)\right)=\alpha z(\xi), \quad E I\left(\varphi^{\prime}\left(\xi_{+}\right)-\varphi^{\prime}\left(\xi_{-}\right)\right)=\beta \psi(\xi) \\
\kappa\left(w^{\prime}(\ell)-\varphi(\ell)\right)=0, \quad E I \varphi^{\prime}(\ell)=0
\end{array}\right.
$$

Solving these equations, we obtain

$$
\begin{aligned}
\varphi(x)=- & \frac{1}{E I}\left[I_{\rho} \int_{0}^{\ell} k(x, s) g_{2}(s) \mathrm{d} s+\rho \int_{0}^{\ell} k(x, s) \mathrm{d} s \int_{s}^{\ell} f_{2}(t) \mathrm{d} t\right. \\
& \left.+\beta g_{1}(\xi) k(\xi, x)+\alpha f_{1}(\xi) \eta(x)\right] \\
w(x)= & \int_{0}^{x} \varphi(s) \mathrm{d} s-\frac{\rho}{\kappa} \int_{0}^{\ell} k(x, s) f_{2}(s) \mathrm{d} s-\frac{\alpha}{\kappa} f_{1}(\xi) k(\xi, x),
\end{aligned}
$$

where

$$
k(x, s)= \begin{cases}s, & 0 \leq s \leq x \\ x, & x \leq s \leq \ell\end{cases}
$$




$$
\eta(x)= \begin{cases}\ell \xi-\frac{1}{2} \xi^{2}, & \xi \leq x \leq \ell, \\ \ell x-\frac{1}{2} x^{2}, & 0 \leq x \leq \xi .\end{cases}
$$

Since the $Y=\left[w, f_{1}, \varphi, g_{1}\right]^{T}$ given above belongs to $\mathcal{D}(\mathcal{A})$ and $Y=\mathcal{A}^{-1} F$, so by the Sobolev Embedding theorem, $\mathcal{A}^{-1}$ is compact and so is the resolvent of $\mathcal{A}$.

Next, for any $Y:=[w, z, \varphi, \psi]^{T} \in \mathcal{D}(\mathcal{A})$, we have

$$
\operatorname{Re}\langle\mathcal{A} Y, Y\rangle=-\alpha|z(\xi)|^{2}-\beta|\psi(\xi)|^{2} \leq 0
$$

from a direct calculation. So $\mathcal{A}$ is dissipative and generates a $C_{0}$ semigroup of contractions by Lumer-Phillips theorem (see [17]) and the last assertion follows accordingly.

Theorem 2.1 tells us that the spectrum of $\mathcal{A}$ are all eigenvalues and so our next task is to determine the eigenvalues of $\mathcal{A}$. Let $\lambda \in \mathcal{C}$ ( $\mathcal{C}$ denotes the complex plane) be an eigenvalue of $\mathcal{A}$, and let $Y=[w, z, \varphi, \psi]^{T} \in$ $\mathcal{D}(\mathcal{A})$ be an associated eigenfunction. Then we have

$$
z(x)=\lambda w(x), \psi(x)=\lambda \varphi(x),
$$

and the function pair $(w(x), \varphi(x))$ satisfying the following equations

$$
\left\{\begin{array}{l}
\rho \lambda^{2} w(x)-\kappa\left(w^{\prime \prime}(x)-\varphi^{\prime}(x)\right)=0, \quad 0<x<\ell, \\
I_{\rho} \lambda^{2} \varphi(x)-E I \varphi^{\prime \prime}(x)-\kappa\left(w^{\prime}(x)-\varphi(x)\right)=0, \quad 0<x<\ell,
\end{array}\right.
$$

with conditions

$$
\left\{\begin{array}{l}
w(0)=0, \quad \varphi(0)=0, \quad w\left(\xi_{+}\right)=w\left(\xi_{-}\right), \quad \varphi\left(\xi_{+}\right)=\varphi\left(\xi_{-}\right), \\
\kappa\left(w^{\prime}\left(\xi_{+}\right)-w^{\prime}\left(\xi_{-}\right)\right)=\alpha \lambda w(\xi), \quad E I\left(\varphi^{\prime}\left(\xi_{+}\right)-\varphi^{\prime}\left(\xi_{-}\right)\right)=\beta \lambda \varphi(\xi), \\
\kappa\left(w^{\prime}(\ell)-\varphi(\ell)\right)=0, \quad E I \varphi^{\prime}(\ell)=0 .
\end{array}\right.
$$

Let $\left(w_{j}(\lambda, x), \varphi_{j}(\lambda, x)\right), j=1,2,3,4$, be the fundamental solutions for (2.2) satisfying

$$
\begin{array}{ll}
\left(w_{1}(\lambda, 0), \varphi_{1}(\lambda, 0)\right)=(1,0), \quad\left(w_{2}(\lambda, 0), \varphi_{2}(\lambda, 0)\right)=(0,1), \\
\left(w_{3}^{\prime}(\lambda, 0), \varphi_{3}^{\prime}(\lambda, 0)\right)=(1,0), \quad\left(w_{4}^{\prime}(\lambda, x), \varphi_{4}^{\prime}(\lambda, 0)\right)=(0,1) .
\end{array}
$$

Then any solution of (2.2) can be expressed as

$$
\left\{\begin{array}{l}
w(\lambda, x)=w(0) w_{1}(\lambda, x)+\varphi(0) w_{2}(\lambda, x)+w^{\prime}(0) w_{3}(\lambda, x)+\varphi^{\prime}(0) w_{4}(\lambda, x), \\
\varphi(\lambda, x)=w(0) \varphi_{1}(\lambda, x)+\varphi(0) \varphi_{2}(\lambda, x)+w^{\prime}(0) \varphi_{3}(\lambda, x)+\varphi^{\prime}(0) \varphi_{4}(\lambda, x),
\end{array}\right.
$$

with $w(\lambda, 0)=w(0), \varphi(\lambda, 0)=\varphi(0), w^{\prime}(\lambda, 0)=w^{\prime}(0), \varphi^{\prime}(\lambda, 0)=\varphi^{\prime}(0)$. Employing (2.3), we see that when $0 \leq x \leq \xi$

$$
\begin{aligned}
& w(\lambda, x)=w^{\prime}(0) w_{3}(\lambda, x)+\varphi^{\prime}(0) w_{4}(\lambda, x), \\
& \varphi(\lambda, x)=w^{\prime}(0) \varphi_{3}(\lambda, x)+\varphi^{\prime}(0) \varphi_{4}(\lambda, x),
\end{aligned}
$$

when $\xi \leq x \leq \ell$,

$$
\begin{aligned}
w(\lambda, x)= & {\left[w^{\prime}(0) w_{3}(\lambda, \xi)+\varphi^{\prime}(0) w_{4}(\lambda, \xi)\right] w_{1}(\lambda, x-\xi)+\left[w^{\prime}(0) \varphi_{3}(\lambda, \xi)+\varphi^{\prime}(0) \varphi_{4}(\lambda, \xi)\right] w_{2}(\lambda, x-\xi) } \\
& +\left[w^{\prime}(0)\left(w_{3}^{\prime}(\lambda, \xi)+\frac{\alpha}{\kappa} \lambda w_{3}(\lambda, \xi)\right)+\varphi^{\prime}(0)\left(w_{4}^{\prime}(\lambda, \xi)+\frac{\alpha}{\kappa} \lambda w_{4}(\lambda, \xi)\right)\right] w_{3}(\lambda, x-\xi) \\
& +\left[w^{\prime}(0)\left(\varphi_{3}^{\prime}(\lambda, \xi)+\frac{\beta}{E I} \lambda \varphi_{3}(\lambda, \xi)\right)+\varphi^{\prime}(0)\left(\varphi_{4}^{\prime}(\lambda, \xi)+\frac{\beta}{E I} \lambda \varphi_{4}(\lambda, \xi)\right)\right] w_{4}(\lambda, x-\xi),
\end{aligned}
$$




$$
\begin{aligned}
\varphi(\lambda, x)= & {\left[w^{\prime}(0) w_{3}(\lambda, \xi)+\varphi^{\prime}(0) w_{4}(\lambda, \xi)\right] \varphi_{1}(\lambda, x-\xi)+\left[w^{\prime}(0) \varphi_{3}(\lambda, \xi)+\varphi^{\prime}(0) \varphi_{4}(\lambda, \xi)\right] \varphi_{2}(\lambda, x-\xi) } \\
& +\left[w^{\prime}(0)\left(w_{3}^{\prime}(\lambda, \xi)+\frac{\alpha}{\kappa} \lambda w_{3}(\lambda, \xi)\right)+\varphi^{\prime}(0)\left(w_{4}^{\prime}(\lambda, \xi)+\frac{\alpha}{\kappa} \lambda w_{4}(\lambda, \xi)\right)\right] \varphi_{3}(\lambda, x-\xi) \\
& +\left[w^{\prime}(0)\left(\varphi_{3}^{\prime}(\lambda, \xi)+\frac{\beta}{E I} \lambda \varphi_{3}(\lambda, \xi)\right)+\varphi^{\prime}(0)\left(\varphi_{4}^{\prime}(\lambda, \xi)+\frac{\beta}{E I} \lambda \varphi_{4}(\lambda, \xi)\right)\right] \varphi_{4}(\lambda, x-\xi),
\end{aligned}
$$

where $\left(w^{\prime}(0), \varphi^{\prime}(0)\right)$ is a pair of non-zero solutions of the following linear equations:

$$
\left\{\begin{array}{l}
w^{\prime}(0) A_{11}(\lambda)+\varphi^{\prime}(0) A_{12}(\lambda)=0 \\
w^{\prime}(0) A_{21}(\lambda)+\varphi^{\prime}(0) A_{22}(\lambda)=0
\end{array}\right.
$$

with

$$
\begin{aligned}
& A_{11}(\lambda):=w_{3}(\lambda, \xi)\left[w_{1}^{\prime}(\lambda, \ell-\xi)-\varphi_{1}(\lambda, \ell-\xi)\right]+\varphi_{3}(\lambda, \xi)\left[w_{2}^{\prime}(\lambda, \ell-\xi)-\varphi_{2}(\lambda, \ell-\xi)\right]+\left[w_{3}^{\prime}(\lambda, \xi)+\frac{\alpha}{\kappa} \lambda w_{3}(\lambda, \xi)\right]\left[w_{3}^{\prime}(\lambda, \ell-\xi)-\varphi_{3}(\lambda, \ell-\xi)\right] \\
&+ {\left[\varphi_{3}^{\prime}(\lambda, \xi)+\frac{\beta}{E I} \lambda \varphi_{3}(\lambda, \xi)\right]\left[w_{4}^{\prime}(\lambda, \ell-\xi)-\varphi_{4}(\lambda, \ell-\xi)\right], } \\
& A_{12}(\lambda):=w_{4}(\lambda, \xi)\left[w_{1}^{\prime}(\lambda, \ell-\xi)-\varphi_{1}(\lambda, \ell-\xi)\right]+\varphi_{4}(\lambda, \xi)\left[w_{2}^{\prime}(\lambda, \ell-\xi)-\varphi_{2}(\lambda, \ell-\xi)\right] \\
&+\left[w_{4}^{\prime}(\lambda, \xi)+\frac{\alpha}{\kappa} \lambda w_{4}(\lambda, \xi)\right]\left[w_{3}^{\prime}(\lambda, \ell-\xi)-\varphi_{3}(\lambda, \ell-\xi)\right] \\
&+\left[\varphi_{4}^{\prime}(\lambda, \xi)+\frac{\beta}{E I} \lambda \varphi_{4}(\lambda, \xi)\right]\left[w_{4}^{\prime}(\lambda, \ell-\xi)-\varphi_{4}(\lambda, \ell-\xi)\right], \\
& A_{21}(\lambda):=w_{3}(\lambda, \xi) \varphi_{1}^{\prime}(\lambda, \ell-\xi)+\varphi_{3}(\lambda, \xi) \varphi_{2}^{\prime}(\lambda, \ell-\xi)+\left[w_{3}^{\prime}(\lambda, \xi)+\frac{\alpha}{\kappa} \lambda w_{3}(\lambda, \xi)\right] \varphi_{3}^{\prime}(\lambda, \ell-\xi) \\
&+\left[\varphi_{3}^{\prime}(\lambda, \xi)+\frac{\beta}{E I} \lambda \varphi_{3}(\lambda, \xi)\right] \varphi_{4}^{\prime}(\lambda, \ell-\xi), \\
&\left.A_{22}(\lambda):=w_{4}(\lambda, \xi) \varphi_{1}^{\prime}(\lambda, \ell-\xi)+\varphi_{4}(\lambda, \xi)\right] \varphi_{2}^{\prime}(\lambda, \ell-\xi)+\left[w_{4}^{\prime}(\lambda, \xi)+\frac{\alpha}{\kappa} \lambda w_{4}(\lambda, \xi)\right] \varphi_{3}^{\prime}(\lambda, \ell-\xi) \\
&+\left[\varphi_{4}^{\prime}(\lambda, \xi)+\frac{\beta}{E I} \lambda \varphi_{4}(\lambda, \xi)\right] \varphi_{4}^{\prime}(\lambda, \ell-\xi) .
\end{aligned}
$$

So all the eigenvalues can be found by finding the zeros of the characteristic determinant $\Gamma(\lambda)$,

$$
\Gamma(\lambda)=\left|\begin{array}{ll}
A_{11}(\lambda) & A_{12}(\lambda) \\
A_{21}(\lambda) & A_{22}(\lambda)
\end{array}\right|,
$$

of the coefficient matrix in (2.5). Altogether we have the following result. 


\section{Theorem 2.2.}

1) Let $\lambda \in \mathcal{C}$. Then $\lambda \in \sigma(\mathcal{A})$ if and only if $\Gamma(\lambda)=0$. In this case, an eigenfunction corresponding to $\lambda$ is given by

$$
Y=[w(\lambda, x), \lambda w(\lambda, x), \varphi(\lambda, x), \lambda \varphi(\lambda, x)]^{T},
$$

where $w(\lambda, x)$ and $\varphi(\lambda, x)$ are determined in (2.4). Furthermore, if $\lambda$ is an eigenvalue for $\mathcal{A}$, then the corresponding eigensubspace is of dimension at most two.

2) The spectrum $\sigma(\mathcal{A})$ of $\mathcal{A}$ distribute symmetrically with respect to the real axis.

Proof. The first assertion is just a summary of the previous discussion. To prove the second assertion, we use the fact that for any $Y \in \mathcal{D}(\mathcal{A})$, its conjugate satisfies $\bar{Y} \in \mathcal{D}(\mathcal{A})$ and $\overline{\mathcal{A} Y}=\mathcal{A} \bar{Y}$. So the second assertion holds.

\section{Asymptotic analysis of the eigenvalues And eigenfunctions of $\mathcal{A}$}

Theorem 2.2 gives us a characterization of all the eigenvalues in terms of the zeros of $\Gamma(\lambda)$ in (2.7).

We now conduct an asymptotic analysis on $\Gamma(\lambda)$ to deduce the corresponding asymptotic behaviour for the eigenvalues. We begin with expanding the eigenfunctions asymptotically, and always assume that

$$
\rho_{1} \neq \rho_{2} \quad \text { and denoted } \quad \rho_{1}^{2}:=\frac{\rho}{\kappa}, \quad \rho_{2}^{2}:=\frac{I_{\rho}}{E I} .
$$

Assume that $\lambda \in \mathcal{C}$ with $|\operatorname{Im} \lambda|$ being large enough and $-M \leq \operatorname{Re} \lambda \leq 0$, in which $M$ is another large positive constant. Then, by expanding asymptotically in $\lambda$, we obtain the following asymptotic expressions:

$$
\left\{\begin{array}{l}
w_{1}(\lambda, x)=\cosh \rho_{1} \lambda x+O\left(\lambda^{-2}\right) \\
\varphi_{1}(\lambda, x)=\frac{-\rho}{E I\left(\rho_{1}^{2}-\rho_{2}^{2}\right)}\left(\frac{\sinh \rho_{1} \lambda x}{\rho_{1} \lambda}-\frac{\sin \rho_{2} \lambda x}{\rho_{2} \lambda}\right)+O\left(\lambda^{-3}\right) \\
w_{2}(\lambda, x)=\frac{\rho_{2}^{2}}{\left(\rho_{1}^{2}-\rho_{2}^{2}\right)}\left(\frac{\sinh \rho_{1} \lambda x}{\rho_{1} \lambda}-\frac{\sin \rho_{2} \lambda x}{\rho_{2} \lambda}\right)+O\left(\lambda^{-3}\right) \\
\varphi_{2}(\lambda, x)=\cosh \rho_{2} \lambda x+O\left(\lambda^{-2}\right) \\
w_{3}(\lambda, x)=\frac{\sinh \rho_{1} \lambda x}{\rho_{1} \lambda}+O\left(\lambda^{-3}\right) \\
\varphi_{3}(\lambda, x)=O\left(\lambda^{-2}\right) \\
w_{4}(\lambda, x)=O\left(\lambda^{-2}\right) \\
\varphi_{4}(\lambda, x)=\frac{\sinh \rho_{2} \lambda x}{\rho_{2} \lambda}+O\left(\lambda^{-3}\right) \\
w_{1}^{\prime}(\lambda, x)=\rho_{1} \lambda \sinh \rho_{1} \lambda x+O\left(\lambda^{-1}\right) \\
\varphi_{1}^{\prime}(\lambda, x)=\frac{-\rho}{E I\left(\rho_{1}^{2}-\rho_{2}^{2}\right)}\left(\cosh \rho_{1} \lambda x-\cosh \rho_{2} \lambda x\right)+O\left(\lambda^{-2}\right) \\
w_{2}^{\prime}(\lambda, x)=\frac{\rho_{2}^{2}}{\left(\rho_{1}^{2}-\rho_{2}^{2}\right)}\left(\cosh \rho_{1} \lambda x-\cosh \rho_{2} \lambda x\right)+O\left(\lambda^{-2}\right) \\
\varphi_{2}^{\prime}(\lambda, x)=\rho_{2} \lambda \sinh \rho_{2} \lambda x+O\left(\lambda^{-1}\right) \\
\left.w_{3}^{\prime}(\lambda, x)=\cosh \rho_{1} \lambda x+O\left(\lambda^{-2}\right)\right) \\
\varphi_{3}^{\prime}(\lambda, x)=O\left(\lambda^{-1}\right) \\
w_{4}^{\prime}(\lambda, x)=O\left(\lambda^{-1}\right) \\
\varphi_{4}^{\prime}(\lambda, x)=\cosh \rho_{2} \lambda x+O\left(\lambda^{-2}\right)
\end{array}\right.
$$

Substituting these expressions into $\Gamma(\lambda)$ in $(2.7)$, we have:

$$
\Gamma(\lambda)=G(\lambda)+O\left(\lambda^{-1}\right),
$$


where the leading term

$$
\begin{aligned}
G(\lambda):= & {\left[\cosh \rho_{1} \lambda \ell+\frac{\alpha}{2 \kappa \rho_{1}} \sinh \rho_{1} \lambda \ell-\frac{\alpha}{2 \kappa \rho_{1}} \sinh \rho_{1} \lambda(\ell-2 \xi)\right]\left[\cosh \rho_{2} \lambda \ell\right.} \\
& \left.+\frac{\beta}{E I \rho_{2}} \sinh \rho_{2} \lambda \ell-\frac{\beta}{2 E I \rho_{2}} \sinh \rho_{2} \lambda(\ell-2 \xi)\right]
\end{aligned}
$$

From expression (3.4), we know that $G(\lambda)$ is analytic in $\mathcal{C}$ and deduce automatically the following remarks.

Remark. (i) When $\xi=\frac{1}{2} \ell$, then $G(\lambda)$ have no zeros in $|\operatorname{Re} \lambda|>M$ for some large enough positive constant $M$. It is because when $\xi=\frac{1}{2} \ell, G(\lambda)$ has a very simple expression:

$$
G(\lambda)=\left[\cosh \rho_{1} \lambda \ell+\frac{\alpha}{2 \kappa \rho_{1}} \sinh \rho_{1} \lambda \ell\right]\left[\cosh \rho_{2} \lambda \ell+\frac{\beta}{E I \rho_{2}} \sinh \rho_{2} \lambda \ell\right]
$$

and so $G(\lambda)$ has no zeros in $|\operatorname{Re} \lambda|>M$ for some large enough positive constant $M$.

(ii) When $\xi \neq \frac{1}{2} \ell$ but $\left(1-\frac{\alpha}{2 \kappa \rho_{1}}\right)=0$ and $\left(1-\frac{\beta}{2 E I \rho_{2}}\right) \neq 0$, we have

$$
G(\lambda) \mathrm{e}^{\rho_{1} \lambda|\ell-2 \xi|+\rho_{2} \lambda \ell} \rightarrow \frac{1}{4} \operatorname{sign}(\ell-2 \xi)\left(1-\frac{\beta}{2 E I \rho_{2}}\right) \quad \text { as } \quad \operatorname{Re} \lambda \rightarrow-\infty
$$

(iii) When $\left(1-\frac{\alpha}{2 \kappa \rho_{1}}\right) \neq 0$ and $\left(1-\frac{\beta}{2 E I \rho_{2}}\right) \neq 0$, we have

$$
G(\lambda) \mathrm{e}^{\left(\rho_{1}+\rho_{2}\right) \lambda \ell} \rightarrow \frac{1}{4}\left(1-\frac{\alpha}{2 \kappa \rho_{1}}\right)\left(1-\frac{\beta}{2 E I \rho_{2}}\right) \quad \text { as } \quad \operatorname{Re} \lambda \rightarrow-\infty .
$$

(iv) As a conclusion of (i-iii), we see that, whenever $\xi \in(0, \ell)$, the zeros of $G(\lambda)$ are located asymptotically in a vertical strip of the complex plane $\mathcal{C}$ centered at the imaginary axis.

We use these remarks and $(3.3,3.4)$ to deduce some asymptotic behaviour for the eigenvalues of $\mathcal{A}$.

Theorem 3.1. With (3.1) and assume that $\left(1-\frac{\alpha}{2 \kappa \rho_{1}}\right) \neq 0$ and $\left(1-\frac{\beta}{2 E I \rho_{2}}\right) \neq 0$. For $n \in Z$, let $\zeta_{n}^{(1)}$ and $\zeta_{n}^{(2)}$ be the roots of respectively the first and the second equation in

$$
\left\{\begin{array}{l}
\cosh \rho_{1} \lambda \ell+\frac{\alpha}{2 \kappa \rho_{1}} \sinh \rho_{1} \lambda \ell-\frac{\alpha}{2 \kappa \rho_{1}} \sinh \rho_{1} \lambda(\ell-2 \xi)=0, \\
\cosh \rho_{2} \lambda \ell+\frac{\beta}{2 E I \rho_{2}} \sinh \rho_{2} \lambda \ell-\frac{\beta}{2 E I \rho_{2}} \sinh \rho_{2} \lambda(\ell-2 \xi)=0 .
\end{array}\right.
$$

Let

$$
\lambda_{n}^{(1)}:=\zeta_{n}^{(1)}+\eta_{n}^{(1)} \quad \text { and } \quad \lambda_{n}^{(2)}:=\zeta_{n}^{(2)}+\eta_{n}^{(2)}
$$

be the eigenvalues of $\mathcal{A}$. Then the following assertions are true:

1) when $|\lambda|$ large enough then $-M \leq \operatorname{Re} \lambda \leq M$ and the degree of the zero $\lambda_{n}^{(j)}$ of $\Gamma(\lambda)$ is the same as that of the zero $\zeta_{n}^{(j)}$ of $G(\lambda)$;

2) the eigenvalues $\lambda_{n}^{(j)} \in \sigma(\mathcal{A})$, with sufficiently large modulus, satisfy $\lambda_{n}^{(j)}-\zeta_{n}^{(j)}=O\left(\frac{1}{\zeta_{n}^{(j)}}\right)$ for $j=1,2$. 
Proof. The first assertion is just a restatement of the above remarks. For $\lambda_{n}^{(j)}:=\zeta_{n}^{(j)}+\eta_{n}^{(j)}(j=1,2)$ to be eigenvalues of $\mathcal{A}$, we must have $\Gamma\left(\lambda_{n}^{(j)}\right)=0$. Thus, for $-M \leq \operatorname{Re} \lambda_{n}^{(j)} \leq M$ with $\left|\operatorname{Im} \lambda_{n}^{(j)}\right|$ large enough, we have

$$
G\left(\lambda_{n}^{(j)}\right)=O\left(\frac{1}{\lambda_{n}^{(j)}}\right)
$$

and so by Rouché theorem, $\eta_{n}^{(j)}=O\left(1 / \zeta_{n}^{(j)}\right)$ for $j=1,2$ and this proves the remaining assertions.

We now ready to estimate the asymptotic behaviour of the eigenfunctions that corresponding to the eigenvalues $\lambda_{n}^{(1)}$ and $\lambda_{n}^{(2)}$. For this, we consider

$$
\left\{\begin{array}{lll}
w^{\prime}(0)=A_{22}\left(\lambda_{n}^{(1)}\right) & \text { and } & \varphi^{\prime}(0)=-A_{21}\left(\lambda_{n}^{(1)}\right), \\
w^{\prime}(0)=A_{12}\left(\lambda_{n}^{(2)}\right) & \text { and } & \varphi^{\prime}(0)=-A_{11}\left(\lambda_{n}^{(2)}\right),
\end{array}\right.
$$

respectively, where $A_{k j}(\lambda)$ are given by $(2.5,2.6)$. Using the estimates in $(3.1)$ and $(3.2)$, we find that

$$
\begin{gathered}
A_{21}(\lambda)=O\left(\lambda^{-1}\right), \quad A_{12}(\lambda)=O\left(\lambda^{-1}\right), \\
A_{22}(\lambda)=\cosh \rho_{2} \ell \lambda+\frac{\beta}{2 E I \rho_{2}} \sinh \rho_{2} \ell \lambda-\frac{\beta}{2 E I \rho_{2}} \sinh \rho_{2} \lambda(\ell-2 \xi)+O\left(\lambda^{-1}\right), \\
A_{11}(\lambda)=\cosh \rho_{1} \ell \lambda+\frac{\alpha}{2 \kappa \rho_{1}} \sinh \rho_{1} \ell \lambda-\frac{\alpha}{2 K \rho_{1}} \sinh \rho_{2} \lambda(\ell-2 \xi)+O\left(\lambda^{-1}\right) .
\end{gathered}
$$

Let the leading terms be

$$
\begin{gathered}
\widehat{A}_{22}(\lambda):=\cosh \rho_{2} \ell \lambda+\frac{\beta}{2 E I \rho_{2}} \sinh \rho_{2} \ell \lambda-\frac{\beta}{2 E I \rho_{2}} \sinh \rho_{2} \lambda(\ell-2 \xi), \\
\widehat{A}_{11}(\lambda):=\cosh \rho_{1} \ell \lambda+\frac{\alpha}{2 \kappa \rho_{1}} \sinh \rho_{1} \ell \lambda-\frac{\alpha}{2 \kappa \rho_{1}} \sinh \rho_{2} \lambda(\ell-2 \xi)
\end{gathered}
$$

and use the estimates (3.2) in some straight-forward but tedious calculations, we obtain the asymptotic expressions for the eigenfunctions of $\mathcal{A}$ :

$$
Y\left(\lambda_{n}^{(1)}\right)=\widehat{A}_{22}\left(\lambda_{n}^{(1)}\right) \Psi\left(\lambda_{n}^{(1)}\right)+Z_{1}\left(\lambda_{n}^{(1)}\right)
$$

with $\left\|Z_{1}(\lambda)\right\|=O\left(\lambda^{-1}\right)$,

$$
\Psi(\lambda):=\left(\begin{array}{c}
\frac{\sinh \rho_{1} \lambda x}{\rho_{1} \lambda} \\
\frac{\sinh \rho_{1} \lambda x}{\rho_{1}} \\
0 \\
0
\end{array}\right)+\chi_{[\xi, \ell]}\left(\begin{array}{c}
\frac{\alpha}{\kappa \rho_{1}} \frac{\sinh \rho_{1} \lambda \xi \sinh \rho_{1} \lambda(x-\xi)}{\rho_{1} \lambda} \\
\frac{\alpha}{\kappa \rho_{1}} \frac{\sinh \rho_{1} \lambda \xi \sinh \rho_{1} \lambda(x-\xi)}{\rho_{1}} \\
0 \\
0
\end{array}\right),
$$

and

$$
Y\left(\lambda_{n}^{(2)}\right)=\widehat{A}_{11}\left(\lambda_{n}^{(2)}\right) \Phi\left(\lambda_{n}^{(2)}\right)+Z_{2}\left(\lambda_{n}^{(2)}\right)
$$


with $\left\|Z_{2}(\lambda)\right\|=O\left(\lambda^{-1}\right)$,

$$
\Phi(\lambda):=\left(\begin{array}{c}
0 \\
0 \\
\frac{\sinh \rho_{2} \lambda x}{\rho_{2} \lambda} \\
\frac{\sinh \rho_{2} \lambda x}{\rho_{2}}
\end{array}\right)+\chi_{[\xi, \ell]}\left(\begin{array}{c}
0 \\
0 \\
\frac{\beta}{E I \rho_{2}} \frac{\sinh \rho_{2} \lambda \xi \sinh \rho_{2} \lambda(x-\xi)}{\rho_{2} \lambda} \\
\frac{\beta}{E I \rho_{2}} \frac{\sinh \rho_{2} \lambda \xi \sinh \rho_{2} \lambda(x-\xi)}{\rho_{2}}
\end{array}\right)
$$

Thus, we can prove the following result.

Theorem 3.2. With (3.1) and assume that $\left(1-\frac{\alpha}{2 \kappa \rho_{1}}\right) \neq 0$ and $\left(1-\frac{\beta}{2 E I \rho_{2}}\right) \neq 0$. Let $\Psi(\lambda)$, $\Phi(\lambda)$ be defined as in (3.7)-(3.8). Let the zeros $\zeta_{n}^{(j)}, \lambda_{n}^{(j)}, j=1,2$, be given as in Theorem 3.1. Then the following assertions are true:

1) an eigenfunction corresponding to $\lambda_{n}^{(1)}$ is given by

$$
\widehat{Y}\left(\lambda_{n}^{(1)}\right):=\Psi\left(\zeta_{n}^{(1)}\right)+\widehat{Z}_{1}\left(\lambda_{n}^{(1)}\right)
$$

with $\left\|\widehat{Z}_{1}(\lambda)\right\|=O\left(\lambda^{-1}\right)$, and an eigenfunction corresponding to $\lambda_{n}^{(2)}$ is given by

$$
\widehat{Y}\left(\lambda_{n}^{(2)}\right):=\Phi\left(\zeta_{n}^{(2)}\right)+\widehat{Z}_{2}\left(\lambda_{n}^{(2)}\right)
$$

with $\left\|\widehat{Z}_{2}(\lambda)\right\|=O\left(\lambda^{-1}\right)$;

2) for each $j=1,2$, the eigenvalues satisfies

$$
\sum_{n \in Z}\left|\lambda_{n}^{(j)}\right|^{-2}<\infty
$$

Proof. From the expressions of $\widehat{A}_{11}$ and $\widehat{A}_{22}$, we first have

$$
\inf _{n \in Z}\left|\widehat{A}_{11}\left(\lambda_{n}^{(1)}\right)\right|>0 \text { and } \inf _{n \in Z}\left|\widehat{A}_{22}\left(\lambda_{n}^{(2)}\right)\right|>0 \text {. }
$$

If we take

and

$$
\widehat{Y}\left(\lambda_{n}^{(1)}\right):=\frac{Y\left(\lambda_{n}^{(1)}\right)}{\widehat{A}_{22}\left(\lambda_{n}^{(1)}\right)}, \quad \widehat{Y}\left(\lambda_{n}^{(2)}\right):=\frac{Y\left(\lambda_{n}^{(2)}\right)}{\widehat{A}_{11}\left(\lambda_{n}^{(2)}\right)}
$$

then

$$
\begin{aligned}
& \widehat{Z}_{1}\left(\lambda_{n}^{(1)}\right):=\Psi\left(\lambda_{n}^{(1)}\right)-\Psi\left(\zeta_{n}^{(1)}\right)+\frac{Z_{1}\left(\lambda_{n}^{(1)}\right)}{\widehat{A}_{22}\left(\lambda_{n}^{(1)}\right)}, \\
& \widehat{Z}_{2}\left(\lambda_{n}^{(2)}\right):=\Phi\left(\lambda_{n}^{(2)}\right)-\Phi\left(\zeta_{n}^{(2)}\right)+\frac{Z_{2}\left(\lambda_{n}^{(2)}\right)}{\widehat{A}_{11}\left(\lambda_{n}^{(2)}\right)}
\end{aligned}
$$

$$
\begin{aligned}
& \widehat{Y}\left(\lambda_{n}^{(1)}\right)=\Psi\left(\zeta_{n}^{(1)}\right)+\widehat{Z}_{1}\left(\lambda_{n}^{(1)}\right) \\
& \widehat{Y}\left(\lambda_{n}^{(2)}\right)=\Phi\left(\zeta_{n}^{(2)}\right)+\widehat{Z}_{2}\left(\lambda_{n}^{(2)}\right) .
\end{aligned}
$$


Also,

$$
\begin{aligned}
\left\|\Psi\left(\lambda_{n}^{(1)}\right)-\Psi\left(\zeta_{n}^{(1)}\right)\right\|^{2}= & \int_{0}^{\ell} \kappa\left[\cosh \rho_{1} \lambda_{n}^{(1)} x-\cosh \rho_{1} \zeta_{n}^{(1)} x+\chi_{[\xi, \ell]} \frac{\alpha}{\kappa \rho_{1}}\left(\sinh \rho_{1} \lambda_{n}^{(1)} \xi \cosh \rho_{1} \lambda_{n}^{(1)}(x-\xi)\right.\right. \\
& \left.\left.-\sinh \rho_{1} \zeta_{n}^{(1)} \xi \cosh \rho_{1} \zeta_{n}^{(1)}(x-\xi)\right)\right]^{2} \mathrm{~d} x \\
& +\int_{0}^{\ell} \frac{\rho}{\rho_{1}^{2}}\left[\sinh \rho_{1} \lambda_{n}^{(1)} x-\sinh \rho_{1} \zeta_{n}^{(1)} x+\chi_{[\xi, \ell]} \frac{\alpha}{\kappa \rho_{1}}\left(\sinh \rho_{1} \lambda_{n}^{(1)} \xi \sinh \rho_{1} \lambda_{n}^{(1)}(x-\xi)\right.\right. \\
& \left.\left.-\sinh \rho_{1} \zeta_{n}^{(1)} \xi \sinh \rho_{1} \zeta_{n}^{(1)}(x-\xi)\right)\right]^{2} \mathrm{~d} x \\
= & \left.\left.O\left(\mid \lambda_{n}^{(1)}-\zeta_{n}^{(1)}\right)\right|^{2}\right)
\end{aligned}
$$

and

$$
\begin{aligned}
\left\|\Phi\left(\lambda_{n}^{(2)}\right)-\Phi\left(\zeta_{n}^{(2)}\right)\right\|^{2}= & \int_{0}^{\ell} \kappa\left[\left(\frac{\sinh \rho_{2} \lambda_{n}^{(2)} x}{\rho_{2} \lambda_{n}^{(2)}}-\frac{\sinh \rho_{1} \zeta_{n}^{(2)} x}{\rho_{2} \zeta_{n}^{(2)}}\right)+\chi_{[\xi, \ell]} \frac{\beta}{E I \rho_{2}}\left(\frac{\sinh \rho_{2} \lambda_{n}^{(2)} \xi \sinh \rho_{2} \lambda_{n}^{(2)}(x-\xi)}{\rho_{2} \lambda_{n}^{(2)}}\right.\right. \\
& \left.\left.-\frac{\sinh \rho_{2} \zeta_{n}^{(2)} \xi \cosh \rho_{2} \zeta_{n}^{(2)}(x-\xi)}{\rho_{2} \zeta_{n}^{(2)}}\right)\right]^{2} \mathrm{~d} x \\
& +\int_{0}^{\ell} E I\left[\cosh \rho_{2} \lambda_{n}^{(2)} x-\cosh \rho_{2} \zeta_{n}^{(2)} x+\chi_{[\xi, \ell]} \frac{\beta}{E I \rho_{2}}\left(\sinh \rho_{2} \lambda_{n}^{(2)} \xi \cosh \rho_{2} \lambda_{n}^{(2)}(x-\xi)\right.\right. \\
& \left.\left.-\sinh \rho_{2} \zeta_{n}^{(2)} \xi \cosh \rho_{2} \zeta_{n}^{(1)}(x-\xi)\right)\right]^{2} \mathrm{~d} x \\
& +\int_{0}^{\ell} \frac{I_{\rho}}{\rho_{2}^{2}}\left[\sinh \rho_{2} \lambda_{n}^{(1)} x-\sinh \rho_{1} \zeta_{n}^{(2)} x+\chi_{[\xi, \ell]} \frac{\beta}{E I \rho_{2}}\left(\sinh \rho_{1} \lambda_{n}^{(2)} \xi \sinh \rho_{1} \lambda_{n}^{(2)}(x-\xi)\right.\right. \\
& \left.\left.-\sinh \rho_{1} \zeta_{n}^{(2)} \xi \sinh \rho_{1} \zeta_{n}^{(2)}(x-\xi)\right)\right]^{2} \mathrm{~d} x \\
= & \left.\left.O\left(\mid \lambda_{n}^{(2)}-\zeta_{n}^{(2)}\right)\right|^{2}\right),
\end{aligned}
$$

so

$$
\left.\left\|\Psi\left(\lambda_{n}^{(1)}\right)-\Psi\left(\zeta_{n}^{(1)}\right)\right\|=O\left(1 / \lambda_{n}^{(1)}\right), \quad\left\|\Phi\left(\lambda_{n}^{(2)}\right)-\Phi\left(\zeta_{n}^{(2)}\right)\right\|=O\left(1 / \lambda_{n}^{(2)}\right)\right),
$$

and therefore 1) is true because $\left\|\widehat{Z}_{1}\left(\lambda_{n}^{(1)}\right)\right\|=O\left(1 / \lambda_{n}^{(1)}\right),\left\|\widehat{Z}_{2}\left(\lambda_{n}^{(2)}\right)\right\|=O\left(1 / \lambda_{n}^{(2)}\right)$.

For 2), since $\Gamma(\lambda)$ is an entire function of finite exponential type, so the assertion follows from the standard result of entire function of finite exponential type ( $c f .[18])$.

We are now in a position to discuss the zeros of $G(\lambda)$ in (3.4). For brevity, denote

$$
\eta:=\rho_{1} \lambda \ell, \quad \zeta:=\rho_{2} \lambda \ell, \quad \xi:=s \ell .
$$

Since

with

$$
G(\lambda)=\widehat{A}_{11}(\lambda) \widehat{A}_{22}(\lambda):=g_{1}(\eta) g_{2}(\zeta)
$$

$$
\left\{\begin{array}{l}
g_{1}(\eta):=\widehat{A}_{11}(\lambda)=\cosh \eta+\frac{\alpha}{2 \kappa \rho_{1}} \sinh \eta+\frac{\alpha}{2 \kappa \rho_{1}} \sinh \eta(2 s-1), \\
g_{2}(\zeta):=\widehat{A}_{22}(\lambda)=\cosh \zeta+\frac{\beta}{2 E I \rho_{2}} \sinh \zeta+\frac{\beta}{2 E I \rho_{2}} \sinh \zeta(2 s-1),
\end{array}\right.
$$


so we only need to study the zeros of

$$
g(z):=\cosh z+K \sinh z+K \sinh (2 s-1) z
$$

where $K$ is a constant and $s \in(0,1)$. Generally speaking, finding the zeros of $g(z)$ is very difficult. However, we have the following assertion.

Theorem 3.3. Let $g(z)$ be defined by (3.13). Assume that $K \neq 1$ and $s \in(0,1)$, then the following assertions are true:

1) there exist constants $A, B$ and $R$ such that for all $|x| \geq R$,

$$
A \mathrm{e}^{|x|} \leq|g(x+i y)| \leq B \mathrm{e}^{|x|}
$$

2) let $\eta_{0}$ be the complex number(s) defined by

$$
\eta_{0}^{2}=\frac{1}{4 s(1-s)}-K^{2}
$$

(i) if $\eta_{0}$ does not solve the following equation (in unknown $\nu$ ):

$$
\left(\frac{\nu \pm \sqrt{\nu^{2}+K^{2}-1}}{1+K}\right)^{(1-2 s)}=\frac{\nu \pm \sqrt{\nu^{2}+K^{2}}}{K},
$$

then all the zeros of the function $g(z)$ are simple and separated;

(ii) if $\eta_{0}$ satisfies equation (3.14), then $s$ must be rational and $\eta_{0}$ is real. Moreover, there exist infinitely many zeros of $g(z)$ with degree at least two.

Proof. 1) Since $g(z)$ is an entire function of exponential type, so when $K \neq 1$, we have

$$
|g(x+i y)| \rightarrow \frac{1}{2}\left\{\begin{array}{l}
(1+K) \mathrm{e}^{x}, \quad \text { as } \quad x \rightarrow+\infty \\
|1-K| \mathrm{e}^{|x|}, \quad \text { as } \quad x \rightarrow-\infty
\end{array}\right.
$$

Hence, there exist $A, B$ and $R$ such that

$$
A \mathrm{e}^{|x|} \leq|g(x+i y)| \leq B \mathrm{e}^{|x|}, \quad \text { when } \quad|x| \geq R
$$

2) (i) To consider the multiplicity of the zeros of $g(z)$, we note that

$$
g^{\prime}(z)=\sinh z+K \cosh z+(2 s-1) K \cosh (2 s-1) z
$$

and

$$
g^{\prime}(z)+g(z)=(1+K) \mathrm{e}^{z}-K \mathrm{e}^{(1-2 s) z}+s K\left[\mathrm{e}^{(1-2 s) z}+\mathrm{e}^{-(1-2 s) z}\right]
$$

Obviously, if $s=\frac{1}{2}, g(z)+g^{\prime}(z)=(1+K) \mathrm{e}^{z} \neq 0$, then all zeros of $g(z)$ are simple and separated. If $s \neq \frac{1}{2}$, denote by $\nu$ the value of $\cosh z+K \sinh z$, i.e.,

$$
\nu:=\cosh z+K \sinh z
$$

then $g(z)=0$ is equivalent to solving the following equations:

$$
\left\{\begin{array}{l}
\cosh z+K \sinh z=\nu \\
K \sinh (1-2 s) z=\nu
\end{array}\right.
$$


Since the first equation is equivalent to

$$
\mathrm{e}^{z}=\frac{\nu \pm \sqrt{\nu^{2}+K^{2}-1}}{1+K}
$$

and the second equation is equivalent to

$$
\mathrm{e}^{(1-2 s) z}=\frac{\nu \pm \sqrt{\nu^{2}+K^{2}}}{K}
$$

so $g(z)=0$ has a solution in $z$ is equivalent to the following equation having a solution in $\nu$ :

$$
\left(\frac{\nu \pm \sqrt{\nu^{2}+K^{2}-1}}{1+K}\right)^{(1-2 s)}=\frac{\nu \pm \sqrt{\nu^{2}+K^{2}}}{K}
$$

which is exactly (3.14). In fact, the solution $z$ and solution $\nu$ are related by

$$
\mathrm{e}^{z}=\frac{\nu \pm \sqrt{\nu^{2}+K^{2}-1}}{1+K}
$$

or

$$
\mathrm{e}^{(1-2 s) z}=\frac{\nu \pm \sqrt{\nu^{2}+K^{2}}}{K}
$$

Now suppose that $\nu$ solves equation (3.14), then $g(z)=0$ and

$$
\begin{aligned}
g^{\prime}(z)+g(z) & =g^{\prime}(z) \\
& =\left(\nu \pm \sqrt{\nu^{2}+K^{2}-1}\right)-\left(\nu \pm \sqrt{\nu^{2}+K^{2}}\right)+2 s\left[\mp \sqrt{\nu^{2}+K^{2}}\right] \\
& = \pm\left(\sqrt{\nu^{2}+K^{2}-1} \pm(2 s-1) \sqrt{\nu^{2}+K^{2}}\right) \\
& = \pm\left[\left(\sqrt{\nu^{2}+K^{2}-1}-\sqrt{\eta_{0}^{2}+K^{2}-1}\right) \pm(2 s-1)\left(\sqrt{\nu^{2}+K^{2}}-\sqrt{\eta_{0}^{2}+K^{2}}\right)\right]
\end{aligned}
$$

because if we set

$$
\eta_{0}^{2}=\frac{1}{4 s(1-s)}-K^{2}
$$

then

$$
\sqrt{\eta_{0}^{2}+K^{2}-1} \pm(2 s-1) \sqrt{\eta_{0}^{2}+K^{2}}=0
$$

Thus, if $\nu \neq \eta_{0}$ then $g^{\prime}(z) \neq 0$, and hence all zeros of $g(z)$ are simple. 
Let $\left\{z_{n}: n \in Z\right\}$ be the set of zeros of $g(z)$, and denote

$$
\nu_{n}:=\cosh z_{n}+K \sinh z_{n}=K \sinh (1-2 s) z_{n} .
$$

Since $z_{n}$ lies in the $\operatorname{strip}-M \leq \operatorname{Re} z \leq M$, so there is a positive $N$ such that $\left|\nu_{n}\right| \leq N$. Let $\nu$ be a cluster point of $\nu_{n}$, then there is a subsequence $\nu_{n_{k}}$ of $\nu_{n}$ such that $\nu_{n_{k}} \rightarrow \nu$. So $\nu$ satisfies the limiting equation. On the other hand, if $\eta_{0}$ does not satisfy equation (3.14) then each $z_{n}$ is a simple zero of $g(z)$. So $g^{\prime}\left(z_{n}\right) \neq 0$. Hence $\inf _{n \in Z}\left|g^{\prime}\left(z_{n}\right)\right|>0$ because if otherwise, there exists a subsequence $\left\{z_{n_{k}}\right\}$ such that $\lim _{k \rightarrow \infty} g^{\prime}\left(z_{n_{k}}\right)=0$, then $\lim _{k \rightarrow \infty} \nu_{n_{k}}=\nu$ and (3.15) imply that $\nu=\eta_{0}$, which is a contradiction. So the zeros of $g(z)$ are separated.

2) (ii) If $\eta_{0}$ solves equation (3.14), we know from the previous arguments that there exists a $z_{0}$ such that $g\left(z_{0}\right)=0, g^{\prime}\left(z_{0}\right)=0$. Therefore

$$
\left\{\begin{array}{l}
\sinh z_{0}+K \cosh z_{0}= \pm \sqrt{\eta_{0}^{2}+K^{2}-1}= \pm \frac{(2 s-1)}{2 \sqrt{s(1-s)}} \\
K \cosh (2 s-1) z_{0}=\mp \sqrt{\eta_{0}^{2}+K^{2}}=\mp \frac{1}{2 \sqrt{s(1-s)}}
\end{array}\right.
$$

Write $z_{0}=x_{0}+i y_{0}$. Notice that the right hands of (3.16) are real and we have

$$
\left\{\begin{array}{l}
\left(\cosh x_{0}+K \sinh x_{0}\right) \sin y_{0}=0 \\
\sinh (2 s-1) x_{0} \sin (2 s-1) y_{0}=0
\end{array}\right.
$$

which imply

$$
\left\{\begin{array}{l}
\sin y_{0}=0 \\
\sin (2 s-1) y_{0}=0
\end{array}\right.
$$

Thus $y_{0}=n \pi$ and $(2 s-1) y_{0}=m \pi$ for some $n, m \in Z$. This shows that $s$ is rational. In this case, we also have

$$
\left\{\begin{array}{l}
0=g\left(z_{0}\right)=\left[\cosh x_{0}+K \sinh x_{0}\right] \cos y_{0}+K \sinh (2 s-1) x_{0} \cos (2 s-1) y_{0}, \\
0=g^{\prime}\left(z_{0}\right)=\left[\sinh x_{0}+K \cosh x_{0}\right] \cos y_{0}+(2 s-1) K \cosh (2 s-1) x_{0} \cos (2 s-1) y_{0} .
\end{array}\right.
$$

Since $g(x)>0$ for $x \geq 0$, so we have $x_{0}<0$ and hence $\eta_{0}$ is a real number.

Write $s:=\frac{q}{p}$, we can take $z_{k}:=z_{0}+i 2 k p \pi$ and we have $(2 s-1) z_{k}=(2 s-1) z_{0}+i k(2 q-p) \pi$ and $g\left(z_{k}\right)=g^{\prime}\left(z_{k}\right)=0$. So $g(z)$ has infinitely many zeros with degree at least two.

We note down a special case.

Corollary 3.4. If $K>1$ and $s \in\left(\frac{1}{2}-\frac{1}{2} \sqrt{1-\frac{1}{K^{2}}}, \frac{1}{2}+\frac{1}{2} \sqrt{1-\frac{1}{K^{2}}}\right)$, then all the zeros of $g(z)$ are simple and separated.

Proof. Let $\eta_{0}$ be defined as in Theorem 3.3. If $\eta_{0}$ satisfies (3.14), then by Theorem 3.3, $\eta_{0}$ is a non-zero real number. This implies that $\frac{1}{4 s(1-s)}-K^{2}>0$, which is

$$
s(1-s)<\frac{1}{4 K^{2}}
$$

or

Therefore

$$
\left|s-\frac{1}{2}\right|>\frac{1}{2} \sqrt{1-\frac{1}{K^{2}}}
$$

$$
s \in\left(0, \frac{1}{2}-\frac{1}{2} \sqrt{1-\frac{1}{K^{2}}}\right) \bigcup\left(\frac{1}{2}+\frac{1}{2} \sqrt{1-\frac{1}{K^{2}}}, 1\right)
$$

because $K>1$ and the required assertion follows from Theorem 3.3.

Putting all the results together, we have the following conclusion on $G(\lambda)$. 
Corollary 3.5. With (3.1) and let $G(\lambda):=\widehat{A}_{11}(\lambda) \widehat{A}_{22}(\lambda)$ (see $\left.(3.11,3.12)\right)$. Set

$$
\xi:=s \ell, \quad K_{1}:=\frac{\alpha}{2 \kappa \rho_{1}}, \quad K_{2}:=\frac{\beta}{2 E I \rho_{2}},
$$

and assume that $\left(1-K_{1}\right) \neq 0$ and $\left(1-K_{2}\right) \neq 0$. Then

1) the zeros of $G(\lambda)$ lie in a vertical strip centered at the imaginary axis and they are given by the roots of (3.5);

2) when $s \in(0,1)$ is irrational, the zeros of $G(\lambda)$ are simple and separated;

3) if $s \in(0,1)$ is rational, then the zeros of $G(\lambda)$ are simple and separated if and only if $\eta_{01}$ and $\eta_{02}$ defined by

$$
\eta_{01}:= \pm \sqrt{\frac{1}{4 s(1-s)}-K_{1}^{2}}, \quad \eta_{02}:= \pm \sqrt{\frac{1}{4 s(1-s)}-K_{2}^{2}}
$$

do not solve (3.14).

\section{RiESZ BASIS PROPERTy OF THE GENERALIZED EIGENFUnCTION SYSTEM OF $\mathcal{A}$}

In this section, we discuss the Riesz basis property of the generalized eigenfunction system of $\mathcal{A}$. Let $K_{j}$ and $\eta_{0 j}, j=1,2$, be defined by (3.17) and (3.18) respectively. Here we always assume that $\left(1-K_{j}\right) \neq 0, j=1,2$, and $\eta_{0 j}, j=1,2$, do not solve equation (3.14). With this assumption, the functions $g_{1}(\eta)$ and $g_{2}(\zeta)$ defined in (3.12) always have infinitely many zeros and were denoted by $\left\{\zeta_{n}^{(1)}: n \in Z\right\}$ and $\left\{\zeta_{n}^{(2)}: n \in Z\right\}$ respectively. According to Theorem 3.2 and the Bari's theorem (see [18], p. 45, Th. 15), if we can prove the Riesz basis property of the sequences $\left\{\Psi\left(\zeta_{n}^{(1)}\right): n \in Z\right\}$ and $\left\{\Phi\left(\zeta_{n}^{(2)}\right): n \in Z\right\}$, then that for the eigenfunctions of $\mathcal{A}$ will also follow. On this regard, we introduce a new norm in $\mathcal{H}$ :

$$
\|Y\|_{1}^{2}:=\int_{0}^{\ell}\left(\kappa\left|w^{\prime}(x)\right|^{2}+\rho|z(x)|^{2}+E I\left|\varphi^{\prime}(x)\right|^{2}+I_{\rho}|\psi(x)|^{2}\right) \mathrm{d} x \quad \text { for } \quad Y=[w, z, \varphi, \psi]^{T} .
$$

Clearly $\|\cdot\|_{1}$ is equivalent to $\|\cdot\|$. Let

$$
\mathcal{H}_{1}:=\left\{Y=[w, z, 0,0]^{T} \in \mathcal{H}\right\}, \quad \mathcal{H}_{2}:=\left\{Y=[0,0, \varphi, \psi]^{T} \in \mathcal{H}\right\}
$$

Under the new norm, we have

$$
\mathcal{H}=\mathcal{H}_{1} \oplus \mathcal{H}_{2}
$$

and we treat $g_{1}$ and $g_{2}$ separately in $\mathcal{H}_{1}$ and $\mathcal{H}_{2}$ in the following two theorems:

Theorem 4.1. Assume that $K_{1} \neq 1$ and $s \in(0,1)$ with $\xi:=s \ell$ such that $g_{1}(\eta)$ defined by (3.12) has simple zeros $\zeta_{n}^{(1)}, n \in Z$. Then the sequence $\left\{\Psi\left(\zeta_{n}^{(1)}\right): n \in Z\right\}$ forms a Riesz Basis in $\mathcal{H}_{1}$.

Proof. Let $\left\{\zeta_{n}^{(1)}: n \in Z\right\}$ be the set of zeros of $g_{1}(\eta)$ and $\Psi\left(\zeta_{n}^{(1)}\right)$ be defined by (3.7). Since $g_{1}(i \eta)$ is an entire function of the sine type, according to Levin Theorem (cf. [18], p. 172, Th. 10), the set $\left\{\mathrm{e}^{\rho_{1} \zeta_{n}^{(1)} x}: n \in Z\right\}$ forms a Riesz basis for $L^{2}[-\ell, \ell]$.

To show that $\left\{\Psi\left(\zeta_{n}^{(1)}\right): n \in Z\right\}$ forms a basis in $\mathcal{H}_{1}$, we introduce some more auxiliary space and operator. Let $H:=V_{0}^{1}[0, \ell] \times L^{2}[0, \ell]$ and define its inner product by

$$
\left\langle Y_{1}, Y_{2}\right\rangle:=\int_{0}^{\ell} \kappa w_{1}^{\prime}(x) \overline{w_{2}^{\prime}(x)} \mathrm{d} x+\int_{0}^{\ell} \rho z_{1}(x) \overline{z_{2}(x)} \mathrm{d} x
$$


for $Y_{1}=\left[w_{1}, z_{1}\right], Y_{2}=\left[w_{2}, z_{2}\right] \in H$. Define an operator $\mathcal{A}_{1}$ in $H$ by:

$$
\mathcal{A}_{1}[w, z]:=\left[z(x), \frac{\kappa}{\rho} w^{\prime \prime}(x)\right]
$$

with domain

$$
\mathcal{D}\left(\mathcal{A}_{1}\right):=\left\{[w, z] \in H \mid w \in V_{0}^{2}(0, \xi) \cup V^{2}(\xi, \ell), \quad z \in V_{0}^{1}(0, \ell), \quad \kappa\left(w^{\prime}\left(\xi_{+}\right)-w^{\prime}\left(\xi_{-}\right)\right)=\alpha z(\xi), \kappa w^{\prime}(\ell)=0\right\}
$$

where $\kappa, \rho, \alpha$ and $\xi \in(0, \ell)$ are the system parameters.

For $\mathcal{A}_{1}$, it is easy to verify that $\zeta_{n}^{(1)}(n \in Z)$ is an eigenvalue with eigenfunction $Y\left(\zeta_{n}^{(1)}\right)$ given by

$$
Y(\lambda):=\left(\sinh \rho_{1} \lambda x+\frac{\alpha}{\kappa \rho_{1}} \chi_{[\xi, \ell]} \sinh \rho_{1} \lambda \xi \sinh \rho_{1} \lambda(x-\xi)\right)\left[\frac{1}{\rho_{1} \lambda}, \frac{1}{\rho_{1}}\right]
$$

It can also be verified directly that $Y^{*}\left(\zeta_{n}^{(1)}\right)$ given by

$$
Y^{*}(\bar{\lambda}):=\left(\sinh \rho_{1} \lambda x+\frac{\alpha}{\kappa \rho_{1}} \chi_{[\xi, \ell]} \sinh \rho_{1} \lambda \xi \sinh \rho_{1} \lambda(x-\xi)\right)\left[\frac{1}{\rho_{1} \lambda},-\frac{1}{\rho_{1}}\right]
$$

is the dual eigenfunction in the sense that $\mathcal{A}_{1}^{*} Y^{*}\left(\zeta_{n}^{(1)}\right)=\overline{\zeta_{n}^{(1)}} Y^{*}\left(\zeta_{n}^{(1)}\right)$, where $\mathcal{A}_{1}^{*}$ is given by

$$
\mathcal{A}_{1}^{*}[w, z]:=-\left[z(x), \frac{\kappa}{\rho} w^{\prime \prime}(x)\right]
$$

with

$\mathcal{D}\left(\mathcal{A}_{1}^{*}\right):=\left\{Y=[w, z] \in H \mid w \in V_{0}^{2}(0, \xi) \cup V^{2}(\xi, \ell), \quad z \in V_{0}^{1}(0, \ell), \kappa\left(w^{\prime}\left(\xi_{+}\right)-w^{\prime}\left(\xi_{-}\right)\right)=-\alpha z(\xi), \kappa w^{\prime}(\ell)=0\right\}$

A direct computation shows that

$$
\left\langle Y\left(\zeta_{n}^{(1)}\right), Y^{*}\left(\zeta_{m}^{(1)}\right)\right\rangle=0, \quad m \neq n, \quad \forall n, m \in Z
$$

and

$$
\left\langle Y\left(\zeta_{n}^{(1)}\right), Y^{*}\left(\zeta_{n}^{(1)}\right)\right\rangle=\kappa \ell+\frac{\alpha}{\kappa \rho_{1}}(\ell-\xi) \sinh 2 \rho_{2} \zeta_{n}^{(1)} \xi+\left(\frac{\alpha}{\kappa \rho_{1}} \sinh \rho_{2} \zeta_{n}^{(1)} \xi\right)^{2}(\ell-\xi) \neq 0
$$


We first show that $\left\{Y\left(\zeta_{n}^{(1)}\right): n \in Z\right\}$ forms a Riesz basis for $H$. Without loss of generality, assume that $\xi \in\left[\frac{1}{2} \ell, \ell\right)$. Then for any $F=[f, g] \in H$, we have

$$
\begin{aligned}
\left\langle F, Y^{*}\left(\zeta_{n}^{(1)}\right)\right\rangle= & \int_{0}^{\ell} \kappa f^{\prime}(x)\left[\cosh \rho_{1} \zeta_{n}^{(1)} x+\chi_{[\xi, \ell]}(x) \frac{\alpha}{2 \kappa \rho_{1}} \sinh \rho_{1} \zeta_{n}^{(1)} x\right. \\
& \left.-\chi_{[\xi, \ell]}(x) \frac{\alpha}{2 \kappa \rho_{1}} \sinh \rho_{1} \zeta_{n}^{(1)}(x-2 \xi)\right] \mathrm{d} x \\
& -\int_{0}^{\ell} \rho \rho_{1}^{-1} g(x)\left[\sinh \rho_{1} \zeta_{n}^{(1)} x+\chi_{[\xi, \ell]}(x) \frac{\alpha}{2 \kappa \rho_{1}} \cosh \rho_{1} \zeta_{n}^{(1)} x\right. \\
& \left.-\chi_{[\xi, \ell]}(x) \frac{\alpha}{2 \kappa \rho_{1}} \cosh \rho_{1} \zeta_{n}^{(1)}(x-2 \xi)\right] \mathrm{d} x \\
= & \frac{1}{2} \int_{0}^{\ell}\left\{\kappa f^{\prime}(x)\left[1+\chi_{[\xi, \ell]}(x) \frac{\alpha}{2 \kappa \rho_{1}}\right]+\frac{\rho}{\rho_{1}} g(x)\left[1+\chi_{[\xi, \ell]}(x) \frac{\alpha}{2 \kappa \rho_{1}}\right]\right. \\
& \left.+\chi_{[2 \xi-\ell, \xi]}(x) \frac{\alpha}{2 \rho_{1}} f^{\prime}(2 \xi-x)+\chi_{[2 \xi-\ell, \xi]}(x) \frac{\alpha}{2} g(2 \xi-x)\right\} \mathrm{e}^{\rho_{1} \zeta_{n}^{(1)} x} \mathrm{~d} x \\
& +\frac{1}{2} \int_{0}^{\ell}\left\{\kappa f^{\prime}(x)\left[1-\chi_{[\xi, \ell]}(x) \frac{\alpha}{2 \kappa \rho_{1}}\right]-\frac{\rho}{\rho_{1}} g(x)\left[1+\chi_{[\xi, \ell]}(x) \frac{\alpha}{2 \kappa \rho_{1}}\right]\right. \\
& \left.-\chi_{[2 \xi-\ell, \xi]}(x) \frac{\alpha}{2 \rho_{1}} f^{\prime}(2 \xi-x)+\chi_{[2 \xi-\ell, \xi]}(x) \frac{\alpha}{2} g(2 \xi-x)\right\} \mathrm{e}^{-\rho_{1} \zeta_{n}^{(1)} x} \mathrm{~d} x \\
= & \int_{-\ell}^{\ell} \mathcal{G}_{F}(x) \mathrm{e}^{\rho_{1} \zeta_{n}^{(1)} x} \mathrm{~d} x,
\end{aligned}
$$

where

$$
\mathcal{G}_{F}(x):=\frac{1}{2}\left\{\begin{array}{c}
\kappa f^{\prime}(x)\left[1+\chi_{[\xi, \ell]}(x) \frac{\alpha}{2 \kappa \rho_{1}}\right]+\frac{\rho}{\rho_{1}} g(x)\left[1+\chi_{[\xi, \ell]}(x) \frac{\alpha}{2 \kappa \rho_{1}}\right] \\
+\chi_{[2 \xi-\ell, \xi]}(x) \frac{\alpha}{2 \rho_{1}} f^{\prime}(2 \xi-x)+\chi_{[2 \xi-\ell, \xi]}(x) \frac{\alpha}{2} g(2 \xi-x), \quad 0 \leq x \leq \ell, \\
\kappa f^{\prime}(x)\left[1-\chi_{[\xi, \ell]}(x) \frac{\alpha}{2 \kappa \rho_{1}}\right]-\frac{\rho}{\rho_{1}} g(x)\left[1+\chi_{[\xi, \ell]}(x) \frac{\alpha}{2 \kappa \rho_{1}}\right] \\
-\chi_{[2 \xi-\ell, \xi]}(x) \frac{\alpha}{2 \rho_{1}} f^{\prime}(2 \xi-x)+\chi_{[2 \xi-\ell, \xi]}(x) \frac{\alpha}{2} g(2 \xi-x), \quad-\ell \leq x<0 .
\end{array}\right.
$$

If for all $n \in Z,\left\langle F, Y^{*}\left(\zeta_{n}^{(1)}\right)\right\rangle=0$, then we have $\mathcal{G}_{F}(x)=0$, a.e. in $[-\ell, \ell]$, i.e., for $0 \leq x \leq(2 \xi-\ell)$,

$$
\left\{\begin{array}{l}
\kappa f^{\prime}(x)+\frac{\rho}{\rho_{1}} g(x)=0 \\
\kappa f^{\prime}(x)-\frac{\rho}{\rho_{1}} g(x)=0
\end{array}\right.
$$

for $(2 \xi-\ell) \leq x \leq \xi$,

$$
\left\{\begin{array}{l}
\kappa f^{\prime}(x)+\frac{\rho}{\rho_{1}} g(x)+\frac{\alpha}{2 \rho_{1}} f^{\prime}(2 \xi-x)+\frac{\alpha}{2} g(2 \xi-x)=0, \\
\kappa f^{\prime}(x)-\frac{\rho}{\rho_{1}} g(x)-\frac{\alpha}{2 \rho_{1}} f^{\prime}(2 \xi-x)+\frac{\alpha}{2} g(2 \xi-x)=0,
\end{array}\right.
$$

for $\xi \leq x \leq \ell$

$$
\left\{\begin{array}{l}
\kappa f^{\prime}(x)\left[1+\frac{\alpha}{2 \kappa \rho_{1}}\right]+\frac{\rho}{\rho_{1}} g(x)\left[1+\frac{\alpha}{2 \kappa \rho_{1}}\right]=0 \\
\kappa f^{\prime}(x)\left[1-\chi_{[\xi, \ell]}(x) \frac{\alpha}{2 \kappa \rho_{1}}\right]-\frac{\rho}{\rho_{1}} g(x)\left[1+\chi_{[\xi, \ell]}(x) \frac{\alpha}{2 \kappa \rho_{1}}\right]=0
\end{array}\right.
$$


Thus, $f^{\prime}(x)=0$ and $g(x)=0$, and hence $F=0$. So the sequence $\left\{Y^{*}\left(\zeta_{n}^{(1)}\right), n \in Z\right\}$ is complete in $H$ and

$$
\sum_{n \in Z}\left|\left\langle F, Y^{*}\left(\zeta_{n}^{(1)}\right)\right\rangle\right|^{2}=\sum_{n \in Z}\left|\left\langle\mathcal{G}_{F}, \mathrm{e}^{\rho_{1} \zeta_{n}^{(1)} x}\right\rangle_{L^{2}}\right|^{2}<\infty
$$

In a similar manner, we can show that the family $\left\{Y\left(\zeta_{n}^{(1)}\right), \in Z\right\}$ is complete in $H$ and

$$
\sum_{n \in Z}\left|\left\langle F, Y\left(\zeta_{n}^{(1)}\right)\right\rangle\right|^{2}<\infty
$$

Hence $\left\{Y\left(\zeta_{n}^{(1)}\right): n \in Z\right\}$ forms a Riesz basis for $H$.

Note that $H=\mathcal{H}_{1}$, in the sense of embedding $H$ into $\mathcal{H}$ isometrically isomorphism, and $Y(\lambda)=\Psi(\lambda)$ with $\Psi(\lambda)$ defined by $(3.7)$, so $\left\{\Psi\left(\zeta_{n}^{(1)}\right): n \in Z\right\}$ forms a Riesz basis and the proof is then complete.

Theorem 4.2. Assume that $K_{2} \neq 1$ and $s \in(0,1)$ with $\xi=$ sl such that $g_{2}(\zeta)$ defined by (3.12) has simple zeros $\zeta_{n}^{(2)}, n \in Z$. Then the sequence $\left\{\Phi\left(\zeta_{n}^{(2)}\right): n \in Z\right\}$ forms a Riesz basis in $\mathcal{H}_{2}$.

Proof. The proof is very similar to that of Theorem 4.1. Let $\left\{\zeta_{n}^{(2)}: n \in Z\right\}$ be the set of zeros of $g_{2}(\zeta)$ and $\Phi\left(\zeta_{n}^{(2)}\right)$ be defined by (3.8). Since $g_{2}(i \zeta)$ is also an entire function of sine type, so the system $\left\{\mathrm{e}^{\rho_{2} \zeta_{n}^{(2)} x}: n \in Z\right\}$ is a Riesz basis for $L^{2}[-\ell, \ell]$.

In order to prove that $\left\{\Phi\left(\zeta_{n}^{(2)}\right): n \in Z\right\}$ forms a basis in $\mathcal{H}_{2}$, similar to the proof of Theorem 4.1, we introduce some auxiliary space and operator.

We equip the space $H=V_{0}^{1}[0, \ell] \times L^{2}[0, \ell]$ with inner product defined by

$$
\left\langle Y_{1}, Y_{2}\right\rangle:=\int_{0}^{\ell} E I \varphi_{1}^{\prime}(x) \overline{\varphi_{2}^{\prime}(x)} \mathrm{d} x+\int_{0}^{\ell} I_{\rho} \psi_{1}(x) \overline{\psi_{2}(x)} \mathrm{d} x
$$

for $Y_{1}=\left[\varphi_{1}, \psi_{1}\right], Y_{2}=\left[\varphi_{2}, \psi_{2}\right] \in H$. Define operator $\mathcal{A}_{2}$ in $H$ by:

$$
\mathcal{A}_{2}[\varphi, \psi]:=\left[\psi(x), \frac{E I}{I_{\rho}} \varphi^{\prime \prime}(x)\right]
$$

with domain

$$
\mathcal{D}\left(\mathcal{A}_{2}\right):=\left\{[w, z] \in H \mid \varphi \in V_{0}^{2}(0, \xi) \cup V^{2}(\xi, \ell), \quad \psi \in V_{0}^{1}(0, \ell), \quad E I\left(\varphi^{\prime}\left(\xi_{+}\right)-\varphi^{\prime}\left(\xi_{-}\right)\right)=\beta \psi(\xi), E I \varphi^{\prime}(\ell)=0\right\}
$$

It is easy to check that $\zeta_{n}^{(2)}, n \in Z$, are eigenvalues of $\mathcal{A}_{2}$ with eigenfunction $Y\left(\zeta_{n}^{(2)}\right)$ defined by

$$
Y(\lambda):=\left(\sinh \rho_{2} \lambda x+\frac{\beta}{E I \rho_{2}} \chi_{[\xi, \ell]} \sinh \rho_{2} \lambda \xi \sinh \rho_{2} \lambda(x-\xi)\right)\left[\frac{1}{\rho_{2} \lambda}, \frac{1}{\rho_{2}}\right]
$$

and $Y^{*}\left(\zeta_{n}^{(2)}\right)$ defined by

$$
Y^{*}(\bar{\lambda}):=\left(\sinh \rho_{1} \lambda x+\frac{\beta}{E I \rho_{2}} \chi_{[\xi, \ell]} \sinh \rho_{2} \lambda \xi \sinh \rho_{2} \lambda(x-\xi)\right)\left[\frac{1}{\rho_{2} \lambda},-\frac{1}{\rho_{2}}\right]
$$

is its dual eigenfunction so that $\mathcal{A}_{2}^{*} Y^{*}\left(\zeta_{n}^{(2)}\right)=\overline{\zeta_{n}^{(2)}} Y^{*}\left(\zeta_{n}^{(2)}\right)$, with $\mathcal{A}_{2}^{*}$ given by

$$
\mathcal{A}_{2}^{*}[\varphi, \psi]:=-\left[\psi(x), \frac{E I}{I_{\rho}} \varphi^{\prime \prime}(x)\right]
$$


and

$\mathcal{D}\left(\mathcal{A}_{2}^{*}\right):=\left\{Y=[\varphi, \psi] \in H \mid \varphi \in V_{0}^{2}(0, \xi) \cup V^{2}(\xi, \ell), \quad \psi \in V_{0}^{1}(0, \ell)\right.$,

$$
\left.E I\left(\varphi^{\prime}\left(\xi_{+}\right)-\varphi^{\prime}\left(\xi_{-}\right)\right)=-\beta \psi(\xi), E I \varphi^{\prime}(\ell)=0\right\}
$$

Using the exact same arguments as in the proof of Theorem 4.1, we can conclude that $\left\{Y\left(\zeta_{n}^{(2)}\right): n \in Z\right\}$ forms a Riesz basis in $H$, and so the sequence $\left\{\Phi\left(\zeta_{n}^{(2)}\right): n \in Z\right\}$ forms a Riesz basis in $\mathcal{H}_{2}$.

Putting these results together, we have the followings.

Corollary 4.3. With (3.1) and let $\Psi\left(\zeta_{n}^{(1)}\right)$ and $\Phi\left(\zeta_{n}^{(2)}\right)$ be given by (3.7) and (3.8) respectively. Assume that $K_{j} \neq 1, j=1,2$, and $s \in(0,1)$ such that $g_{j}, j=1,2$, has simple zeros $\zeta_{n}^{(j)}$ with $n \in Z$. Then the system $\left\{\Psi\left(\zeta_{n}^{(1)}\right), \Phi\left(\zeta_{n}^{(2)}\right): n \in Z\right\}$ forms a Riesz basis for $\mathcal{H}$.

Theorem 4.4. With (3.1) and let $\mathcal{H}, \mathcal{A}, K_{j}, g_{j}, j=1,2$, be defined as before. Assume that $K_{j} \neq 1, j=1,2$, and $s \in(0,1)$ with $\xi=$ sl such that $g_{j}, j=1,2$, has simple zeros $\zeta_{n}^{(j)}$ with $n \in Z$. Then the following assertions are true:

1) the eigenvalues of $\mathcal{A}$ with large enough modulii are algebraically simple;

2) the generalized eigenfunction system of $\mathcal{A}$ forms a Riesz basis of $\mathcal{H}$, and so the spectrum determined growth condition is true for system (2.1).

Proof. The first assertion 1) follows from Theorem 3.1. The second assertion 2) is a consequence of Theorem 3.1 and the Bari's theorem (see [18], p. 45, Th. 15) because the spectrum determined growth condition always holds for a Riesz system, so the proof is complete.

\section{Stability of the Closed loop SyStem}

We now deduce some interesting results on the stabilities of the closed loop system (1.1,1.2). It is amazing to see how the stabilities depend on the location of $\xi$ in $(0, \ell)$. Recall that the system $(1.1,1.2)$ will be a Riesz system whenever the hypotheses of Theorem 4.4 are fulfilled, and so the spectrum determined growth condition is true. Hence, if there exists no eigenvalue of $\mathcal{A}$ on the imaginary axis, then the closed loop system is stable because $\mathcal{A}$ is dissipative ( $c f$. Th. 2.1). Furthermore, if the imaginary axis is not an asymptote for $\sigma(\mathcal{A})$, then the closed loop system is exponentially stable. Therefore, we shall focus on the cases that the imaginary axis contains eigenvalue(s) of $\mathcal{A}$ or is an asymptote for $\sigma(\mathcal{A})$.

Lemma 5.1. Let $G(\lambda)$ be defined in (3.4) and $\xi:=s \ell$ with $s \in(0,1)$.

1) If $s=\frac{2 k}{2 n+1}$ for some integers $k$ and $n$, then the imaginary axis is an asymptote of $\sigma(\mathcal{A})$.

2) On the other hand, if $G(\lambda)$ has a zero on the imaginary axis, then $s=\frac{2 k}{2 n+1}$ for some integers $k$ and $n$.

Proof. 1) From Theorem 3.1, we know that we can use the zeros of $G(\lambda)$ to approximate the eigenvalues of $\mathcal{A}$ when their modulii are large enough. So we only need to prove that there are infinite number of zeros of $G(\lambda)$ on the imaginary axis when $s=\frac{2 k}{2 n+1}$. For these $s$, we take $\eta_{m}$ and $\zeta_{m}$ such that

$$
\eta_{m}:=\rho_{1} \lambda \ell=i(m \pi+\pi / 2) \quad \text { and } \quad \zeta_{m}:=\rho_{2} \lambda \ell=i(m \pi+\pi / 2) .
$$


Then for $m=n+2 \mu(2 n+1), \mu \in Z$, we have

$$
\begin{aligned}
g_{1}\left(\eta_{m}\right) & =\cos (m \pi+\pi / 2)+\frac{\alpha}{2 \kappa \rho_{1}} \sin (m \pi+\pi / 2)-\frac{\alpha}{2 \kappa \rho_{1}} \sin (m \pi+\pi / 2)(1-2 s) \\
& =\frac{\alpha}{2 \kappa \rho_{1}}\left[(-1)^{n}-\sin \left((n \pi+2 \mu(2 n+1) \pi+\pi / 2) \frac{2 n+1-4 k}{2 n+1}\right)\right] \\
& =\frac{\alpha}{2 \kappa \rho_{1}}\left[(-1)^{n}-\sin \left(\frac{(2 n+1-4 k) \pi}{2}+2 \mu(2 n+1-4 k) \pi\right)\right]=0
\end{aligned}
$$

and

$$
g_{2}\left(\zeta_{m}\right)=\cos (m \pi+\pi / 2)+\frac{\beta}{2 E I \rho_{2}} \sin (m \pi+\pi / 2)-\frac{\beta}{2 E I \rho_{2}} \sin (m \pi+\pi / 2)(1-2 s)=0 .
$$

This shows that there are infinitely many zeros of $G(\lambda)=g_{1}(\eta) g_{2}(\zeta)$ on the imaginary axis. So the imaginary axis is an asymptote of $\sigma(\mathcal{A})$ by Theorem 3.1 .

2) Conversely, assume that there is a zero of $G(\lambda)$ on the imaginary axis and let the zero be $\lambda:=i y$, then either $g_{1}(\eta)=0$ or $g_{2}(\zeta)=0$ with $\eta=i \rho_{1} y \ell$ and $\zeta=i \rho_{2} y \ell$. When $g_{1}(\eta)=0$, i.e.,

$$
\cos \rho_{1} y \ell+i \frac{\alpha}{2 \kappa \rho_{1}}\left[\sin \rho_{1} y \ell-\sin \rho_{1} y(1-2 s) \ell\right]=0
$$

we have

$$
\cos \rho_{1} y \ell=0, \quad \sin \rho_{1} y \ell-\sin (1-2 s) \rho_{1} y \ell=0 .
$$

Expanding $\sin (1-2 s) \rho_{1} y \ell$, we have

$$
\cos \rho_{1} y \ell=0 \quad \text { and } \quad 1=\cos 2 s \rho_{1} y \ell
$$

because $\sin \rho_{1} y \ell \neq 0$ when $\cos \rho_{1} y \ell=0$ and there are integers $n$ and $k$ such that

$$
\rho_{1} y \ell=(n \pi+\pi / 2), \quad 2 s \rho_{1} y \ell=2 k \pi .
$$

Hence,

$$
s=\frac{2 k}{2 n+1}
$$

which is also the case when $g_{2}(\zeta)=0$.

Definition 5.2. Denote by $S_{0}$ the set

$$
S_{0}:=\left\{s \in(0,1) \mid s=\frac{2 k}{2 n+1}, n, k \in Z\right\}
$$

and called the points in $S_{0}$ the nodes of the system.

Denote by $\widetilde{S_{0}}$ the set

$\widetilde{S_{0}}:=\left\{s \in(0,1) \mid s \in S_{0}\right.$ or there are $s_{j}:=\frac{2 k(j)}{2 n(j)+1} \in S_{0}$ such that

$$
\left.\lim _{j \rightarrow \infty} s_{j}=s \text { and } \lim _{j \rightarrow \infty}(2 n(j)+1)\left(s-s_{j}\right)=0\right\} .
$$

We now examine some interesting dynamics of the system. 
Remark. It is interesting to note that for each $s \in(0,1), s$ can only be one of the four types: an irrational number, even/odd, odd/odd, odd/even because the case of even/even will reduce to one of the last three cases after factorizations. Easy to see that

$$
(0,1) \backslash S_{0}=\{\text { odd } / \text { odd }\} \cup\{\text { odd/even }\} \cup\{\text { irrationals }\} \cap(0,1) .
$$

Also, we have

$$
\overline{S_{0}} \cap(0,1)=(0,1)
$$

which has a very neat geometric proof. Note that each $s \in S_{0}$ can be viewed as a line with slope $s$ in the $x y$-plane with $(n, k)$ being the integer lattice points. Now for each $x \in(0,1)$ and for $\delta>0$, there always exists some integer lattice points $(n, k)$ between the lines that corresponding to $x+\delta$ and $x-\delta$. Thus there exists an $s$ so that $|x-s| \leq \delta$ and so $\overline{S_{0}}=(0,1)$.

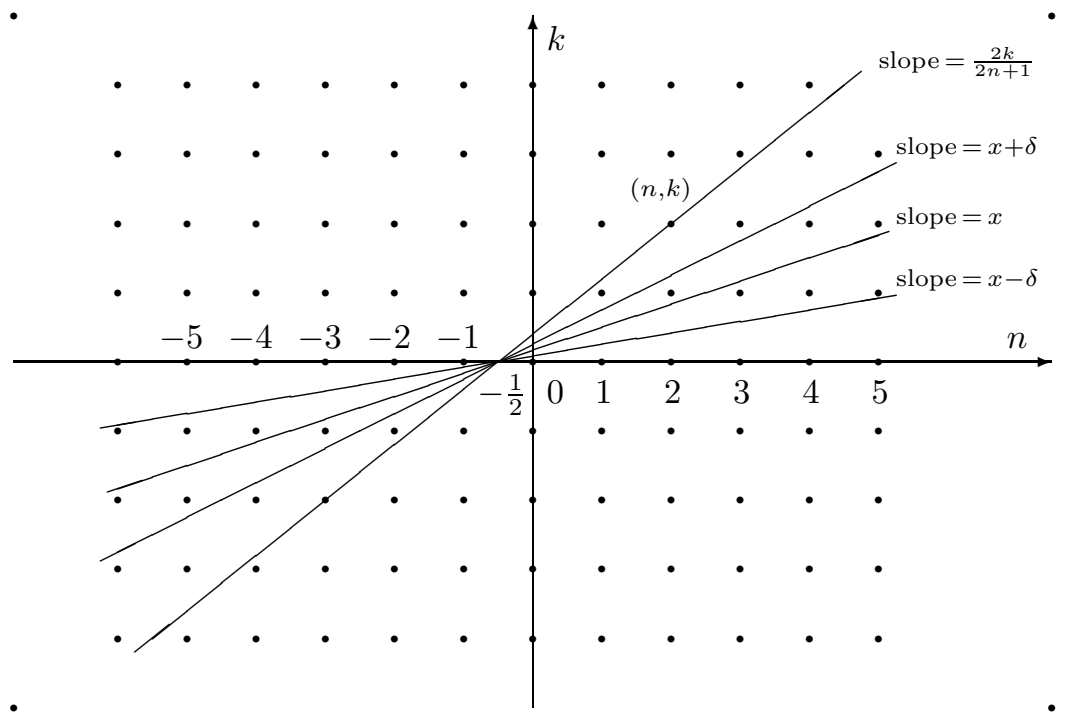

We also have the followings.

Lemma 5.3. $\left\{\frac{p}{q} \in(0,1): p\right.$ is odd and relatively prime to integer $\left.q\right\} \notin \widetilde{S_{0}}$.

Proof. If we can show that all rational $s:=\frac{2 p+1}{q} \in(0,1)$ with integers $p, q$ so that $2 p+1$ and $q$ are relatively prime do not belong to $\widetilde{S_{0}}$, then the assertion holds true.

Now for $s:=\frac{2 p+1}{q} \in(0,1)$, for any $s_{j}:=\frac{2 k(j)}{2 n(j)+1} \in S_{0}$ we have

$$
\left|s_{j}-s\right|(2 n+1)=\left|\frac{2 p+1}{q}-\frac{2 k}{2 n+1}\right|(2 n+1) \geq \frac{1}{q(2 n+1)}(2 n+1)=\frac{1}{q}
$$

because the difference of two distinct integers is at least 1 . Hence, $s$ does not belong to $\widetilde{S_{0}}$.

Remark. Note that for each $\lambda \in \sigma(\mathcal{A})$ and its corresponding eigenfunction

$$
Y=(w(\lambda, x), \lambda w(\lambda, x), \varphi(\lambda, x), \lambda \varphi(\lambda, x))
$$

we have

$$
\frac{1}{2} \frac{\mathrm{d}}{\mathrm{d} t}\langle Y, Y\rangle=\operatorname{Re}\langle\mathcal{A} Y, Y\rangle=\operatorname{Re} \lambda|| Y \|^{2}=-\alpha|\lambda w(\lambda, \xi)|^{2}-\beta|\lambda \varphi(\lambda, \xi)|^{2} \leq 0
$$


So for $s \in S_{0}$, if some eigenvalue $\lambda$ is purely imaginary or the point $\xi=s \ell$ make $w(\lambda, \xi)=\varphi(\lambda, \xi)=0$ for some eigenvalue $\lambda$, then the system is unstable. If $|w(\lambda, \xi)|+|\varphi(\lambda, \xi)| \neq 0$ for all $\lambda \in \sigma(\mathcal{A})$, then $\operatorname{Re} \lambda<0$, for all $\lambda \in \sigma(\mathcal{A})$, and hence the system is asymptotically stable. This stability can be improved to exponential stability if $s \in(0,1) \backslash \widetilde{S_{0}}$. Here are the details.

Theorem 5.4. Under (3.1) and let $\mathcal{H}, \mathcal{A}, K_{j}(j=1,2)$, be defined as before. Assume that $K_{j} \neq 1, j=1,2$, and $s \in(0,1) \backslash \widetilde{S_{0}}$ such that $g_{j}(j=1,2)$ has simple zeros $\zeta_{n}^{(j)}, n \in Z$. Then the system (2.1) is exponentially stable.

Proof. We only need to show that under the assumptions, we have $\inf _{y \in \mathbb{R}}|G(i y)|>0$. In fact, we can show that $\inf _{y \in \mathbb{R}}|G(i y)|=0$ implies that $s \in \widetilde{S_{0}}$.

Suppose that $\inf _{y \in \mathbb{R}}|G(i y)|=0$. Then there exists a sequence $\left\{y_{n}\right\}$ such that

$$
\lim _{n \rightarrow \infty}\left|G\left(i y_{n}\right)\right|=0 \quad \text { and } \quad \lim _{n \rightarrow \infty}\left|y_{n}\right|=\infty
$$

We can assume without loss of generality that $\lim _{n \rightarrow \infty} g_{1}\left(\eta_{n}\right)=0$ with $\eta_{n}:=i \rho_{1} y_{n} \ell$. It implies that

$$
\cos \rho_{1} y_{n} \ell \rightarrow 0 \text { when } n \rightarrow \infty, \quad \text { and } \quad \sin \rho_{1} y_{n} \ell-\sin \rho_{1} y_{n} \ell(1-2 s) \rightarrow 0 \text { when } n \rightarrow \infty .
$$

Hence there exists a sequence of integers $\left\{k_{n}\right\}$ such that $\delta_{1, n}:=\rho_{1} y_{n} \ell-\left(k_{n} \pi+\pi / 2\right) \rightarrow 0$. Since we have

$$
\sin \rho_{1} y_{n} \ell-\sin \rho_{1} y_{n} \ell(1-2 s)=\sin \rho_{1} y_{n} \ell\left(1-\cos 2 \rho_{1} y_{n} \ell s\right)-\cos \rho_{1} y_{n} \ell \sin 2 \rho_{1} y_{n} \ell s
$$

So

$$
\cos 2 \rho_{1} y_{n} \ell s \rightarrow 1
$$

and there exist a sequence of integers $\left\{\eta_{n}\right\}$ such that $\delta_{2, n}:=2 s \rho_{1} y_{n} \ell-2 \eta_{n} \pi \rightarrow 0$. Thus we have

$$
s=\frac{2 \eta_{n}}{2 k_{n}+1}-\frac{1}{2 k_{n}+1}\left[\frac{4 \eta_{n} \delta_{1, n}}{\left(2 k_{n}+1\right) \pi+2 \delta_{1, n}}-\frac{(2 k-n+1) \delta_{2, n}}{\left(2 k_{n}+1\right) \pi+2 \delta_{1, n}}\right] .
$$

So

$$
\lim _{n \rightarrow \infty}\left(2 k_{n}+1\right)\left[s-\frac{2 \eta_{n}}{2 k_{n}+1}\right]=0
$$

and $s \in \widetilde{S_{0}}$. Therefore, we have $\inf _{y \in \mathbb{R}}|G(i y)|>0$ when $s \in(0,1) \backslash \widetilde{S_{0}}$ because $\mathcal{A}$ is dissipative. Hence, the imaginary axis is not an asymptote of $\sigma(\mathcal{A})$.

Also, under the assumptions of this theorem, system (2.1) will be a Riesz system according to Theorem 4.4, and satisfies the spectrum determined growth condition. Hence the system is exponentially stable whenever the spectrum has a positive distance from the imaginary axis, which is true because $s \in(0,1) \backslash \widetilde{S_{0}}$. 
Proof. As a conclusion, we have the following remark:

Remark. Let $K_{j} \neq 1, j=1,2$. For any $s \in(0,1)$, there are several cases:

1) $s \in \widetilde{S_{0}}$. In this cases if there is a $\lambda \in \sigma(\mathcal{A})$ such that $|w(\lambda, \xi)|+|\varphi(\lambda, \xi)|=0$, with $\xi=s \ell$, the system (2.1) is unstable. If $|w(\lambda, \xi)|+|\varphi(\lambda, \xi)| \neq 0, \forall \lambda \in \sigma(\mathcal{A})$, with $\xi=s \ell$, then the system is asymptotically stable;

2) $s \in(0,1) \backslash \widetilde{S_{0}}$. If $\eta_{01}, \eta_{02}$ defined by (3.18) do not solve (3.14), then we know from Theorem 3.5 that the zeros of $G(\lambda)$ are simple and separated. Therefore the conditions of Theorem 5.3 are fulfilled and system (2.1) is a Riesz system and is exponentially stable. In particular, $s=\frac{1}{2}$ belongs this case;

3 ) if one of $\eta_{0 j}, j=1,2$ solves (3.14), then the system (2.1) has infinitely many eigenvalues of multiplicity at least two, and we shall study this degenerate case in another paper.

The authors would like to thank the referees for their useful and helpful comments and suggestions. We are very grateful to Dr. S. K. Chung for his contribution in Lemma 5.3 as well as many other fruitful discussions.

\section{REFERENCES}

[1] G. Chen, C.M. Delfour, A.M. Krall and G. Payre, Modeling, stabilization and control of serially connected beam. SIAM J. Control Optim. 25 (1987) 526-546.

[2] G. Chen, S.G. Krantz, D.W. Ma, C.E. Wayne and H.H. West, The Euler-Bernoulli beam equation with boundary energy dissipation, in Operator methods for optimal control problems, edited by Sung J. Lee. Marcel Dekker, New York (1988) 67-96.

[3] G. Chen, S.G. Krantz, D.L. Russell, C.E. Wayne and H.H. West, Analysis, design and behavior of dissipative joints for coupled beams. SIAM J. Appl. Math. 49 (1989) 1665-1693.

[4] F. Conrad, Stabilization of beams by pointwise feedback control. SIAM J. Control Optim. 28 (1990) 423-437.

[5] J.E. Lagnese, G. Leugering and E. Schmidt, Modeling, analysis and control of dynamic Elastic Multi-link structures. Birkhauser, Basel (1994).

[6] R. Rebarber, Exponential stability of coupled beam with dissipative joints: A frequency domain approach. SIAM J. Control Optim. 33 (1995) 1-28.

[7] K. Ammari and M. Tucsnak, Stabilization of Bernoulli-Euler beams by means of a pointwise feedback force. SIAM J. Control Optim. 39 (2000) 1160-1181.

[8] J.U. Kim and Y. Renardy, Boundary control of the Timoshenko beam. SIAM. J. Control Optim. 25 (1987) 1417-1429.

[9] K. Ito and N. Kunimatsu, Semigroup model and stability of the structurally damped Timoshenko beam with boundary inputs. Int. J. Control 54 (1991) 367-391.

[10] Ö. Morgül, Boundary control of a Timoshenko beam attached to a rigid body: Planar motion. Int. J. Control 54 (1991) 763-791.

[11] D.H. Shi and D.X. Feng, Feedback stabilization of a Timoshenko beam with an end mass. Int. J. Control 69 (1998) $285-300$.

[12] D.X. Feng, D.H. Shi and W.T. Zhang, Boundary feedback stabilization of Timoshenko beam with boundary dissipation. Sci. China Ser. A 41 (1998) 483-490.

[13] F. Conrad and Ö. Morgül, On the stabilization of a flexible beam with a tip mass. SIAM J. Control Optim. 36 (1998) 1962-1986.

[14] B.Z. Guo and R.Y. Yu, The Riesz basis property of discrete operators and application to a Euler-Bernoulli beam equation with boundary linear feedback control. IMA J. Math. Control Inform. 18 (2001) 241-251.

[15] B.P. Rao, Optimal energy decay rate in a damped Rayleigh beam, edited by S. Cox and I. Lasiecka. Contemp. Math. 209 (1997) 221-229.

[16] G.Q. Xu, Boundary feedback control of elastic beams, Ph.D. Thesis. Institute of Mathematics and System Science, Chinese Academy of Sciences (2000).

[17] A. Pazy, Semigroups of linear operators and applications to partial differential equations. Springer-Verlag, New York, Appl. Math. Sci. 44 (1983).

[18] R.M. Young An introduction to nonharmonic Fourier series. Academic Press, Inc. New York (1980). 